\title{
REGULARITY SCALABLE IMAGE CODING BASED ON WAVELET SINGULARITY DETECTION
}

\author{
CHARLOTTE YUK-FAN HO \\ School of Mathematical Sciences, Queen Mary College, University of London. \\ Mile End Road, London, E1 4NS, United Kingdom. \\ Email: c.ho@qmul.ac.uk \\ TAI-CHIU HSUNG \\ Department of Electronic and Information Engineering, Hong Kong Polytechnic University. \\ Hung Hom, Kowloon, Hong Kong, China. \\ Email:entchsung@polyu.edu.hk \\ DANIEL PAK-KONG LUN \\ Department of Electronic and Information Engineering, Hong Kong Polytechnic University. \\ Hung Hom, Kowloon, Hong Kong, China. \\ Email: enpklun@polyu.edu.hk \\ BINGO WING-KUEN LING $\dagger$ \\ Department of Electronic Engineering, King's College London. \\ Strand, London, WC2R 2LS, United Kingdom. \\ Email:wing-kuen.ling@kcl.ac.uk \\ PETER KWONG-SHUN TAM \\ Department of Electronic and Information Engineering, Hong Kong Polytechnic University. \\ Hung Hom, Kowloon, Hong Kong, China. \\ Email: enptam@polyu.edu.hk \\ WAN-CHI SIU \\ Department of Electronic and Information Engineering, Hong Kong Polytechnic University. \\ Hung Hom, Kowloon, Hong Kong, China. \\ Email: enwcsiu@polyu.edu.hk \\ Received (24 January, 2006.) \\ Revised (3 October, 2007.) \\ Accepted (8 October, 2007.)
}

In this paper, we propose an adaptive algorithm for scalable wavelet image coding, which is based on the general feature, the regularity, of images. In pattern recognition or computer vision, regularity of images is estimated from the oriented wavelet coefficients and quantified by the Lipschitz exponents. To estimate the Lipschitz exponents, evaluating the interscale evolution of the wavelet transform modulus sum (WTMS) over the directional cone of influence was proven to be a better approach than tracing the wavelet transform modulus maxima (WTMM). This is because the irregular sampling nature of the WTMM complicates the reconstruction process. Moreover, examples were found to show that the WTMM representation cannot uniquely 


\begin{abstract}
characterize a signal. It implies that the reconstruction of signal from its WTMM may not be consistently stable. Furthermore, the WTMM approach requires much more computational effort. Therefore, we use the WTMS approach to estimate the regularity of images from the separable wavelet transformed coefficients. Since we do not concern about the localization issue, we allow the decimation to occur when we evaluate the interscale evolution. After the regularity is estimated, this information is utilized in our proposed adaptive regularity scalable wavelet image coding algorithm. This algorithm can be simply embedded into any wavelet image coders, so it is compatible with the existing scalable coding techniques, such as the resolution scalable and signal-to-noise ratio (SNR) scalable coding techniques, without changing the bitstream format, but provides more scalable levels with higher peak signal-to-noise ratios (PSNRs) and lower bit rates. In comparison to the other feature-based wavelet scalable coding algorithms, the proposed algorithm outperforms them in terms of visual perception, computational complexity and coding efficiency.
\end{abstract}

Keywords: Feature-based scalable coding; regularity; separable wavelet transform.

\title{
1. Introduction
}

With the decreasing cost and increasing computational power and storage capabilities of digital multimedia systems, more and more visual information and large image databases will be available on-line. For rapid transmission or fast image browsing, it is desirable to provide a fast coarse approximation of the shape of an image at very low bit rates, and then progressively enhance it as more bits are transmitted ${ }^{1}$. This fascinates numerous signal processing researchers to develop new scalable image coding algorithms for such multimedia applications.

Existing wavelet image compression algorithms, such as embedded zero-tree coding of wavelet coefficients (EZW) algorithm ${ }^{2}$ in MPEG-4, set partitioning in hierarchical trees (SPIHT) ${ }^{3}$ and embedded block coding with optimized truncation of the embedded bit streams (EBCOT) algorithms ${ }^{4}$ in JPEG2000, provide scalabilities in both resolution and SNR (or accuracy). Resolution scalability allows different resolutions or sizes of a decoded image to be displayed, which affects the bit rates. SNR or accuracy scalability allows different image quality to be reconstructed by transmitting different numbers of significant bit-planes. However, these two scalabilities only allow a coarse-to-fine approximation of images at various bit rates, which do not exploit the visual perception. Particularly, they do not allow the display of the shape or some features of a decoded image at very low bit rates.

Therefore, various researchers seek for other scalabilities that utilize visually important information of an image. Edges, boundaries, textures and surfaces of an image are usually considered to be the visually important features. So various progressive or scalable wavelet image coding algorithms based on the edges and surfaces of an image were proposed $d^{5-7}$. Nevertheless, these algorithms do not consider some practical issues, such as the computational complexity and coding efficiency. The details will be discussed in Section 2.

The lack of scalable image coding schemes which consider the visual perception, computational complexity and coding efficiency motivates us to develop a simple and effective scalable wavelet image coding algorithm. First of all, we need to determine the type of features that can mostly characterize an image. Then we need to detect and represent these visually important features. Finally, we need to capture these features into the existing wavelet image coders, which is the most important part for the image 
coding application. For the first part, the local visual smoothness of an image is chosen as the feature that characterizes an image. For the second part, as multiscale edges of an image in computer vision or pattern recognition systems are often detected by oriented wavelets with partial derivative of order one ${ }^{8-9}$, and the Lipschitz regularity of an image is related to the asymptotic decay of the wavelet transformed coefficients in the corresponding neighborhood in both horizontal and vertical directions, Lipschitz regularity is used to detect multiscale edges and quantify the local visual smoothness of an image. Since the decay of the wavelet transformed coefficients is controlled by the WTMM $^{10-11}$, it was shown analytically in ${ }^{10}$ that the local WTMM provide numerical information to compute Lipschitz exponents. Nevertheless, many errors and ambiguities are introduced when tracing the WTMM at coarse scales, These include: 1) the irregular sampling nature of the WTMM complicates the reconstruction process, 2) examples were found to show that the WTMM representation cannot uniquely characterize a signal, 3) the reconstruction of signal from its WTMM may not be consistently stable, and 4) the WTMM approach requires much more computational effort. Therefore, the WTMS approach was proposed ${ }^{12}$ to replace tracing the WTMM one. In our scalable coding algorithm, we estimate the Lipschitz regularity from the separable wavelet transformed coefficients by evaluating the interscale evolution of the magnitude sum over the cone of influence. The details of this part will be discussed in Section 3. For the last part, we select the wavelet coefficients at various Lipschitz regularity levels and encode them in the order of starting from the lowest to the highest regularity. Since it is not necessary to perform edge detection, the approximation at the coarse scales after the decimation is still valid and this approach can be embedded into any existing wavelet scalable image coder. The validity under the decimation domain will be demonstrated in our simulation results and the details of this part will be discussed in Sections 4 and 5.

When this scalable coding algorithm is applied in the resolution scalability, we introduce some quality levels at each resolution level. These quality levels are determined from the Lipschitz regularity. We can produce images with higher quality at each resolution level. That means at the same resolution level, it is possible to produce images at much lower bit rates with an insignificant degradation of image quality. Comparing to the existing feature-based scalable wavelet image coding algorithms, the decoded images are better in visual quality and higher in PSNR at similar bit rates. The implementation complexity is also lower among the existing feature-based scalable wavelet image coding algorithms. These results will be shown in Section 5 .

This paper is organized as follows. In Section 2, we review and discuss the existing scalable wavelet coding algorithms based on image features. In Section 3, we review the estimation of the Lipschitz regularity via the WTMS approach. In Section 4, we propose a simple and efficient adaptive regularity scalable coding algorithm, which can be embedded to any existing wavelet image coders. This part is the main contribution of the paper. Section 5 presents the results of the proposed regularity scalable coding algorithm when it is applied in addition to the resolution scalability. The comparison between our results and that of two existing scalable wavelet image coding algorithms, which are based on some features of the image, is also shown in Section 5. Finally, we conclude our works in Section 6. 


\section{Review of the Existing Feature-based Scalable Wavelet Image Coding Algorithms}

For fast image browsing and retrieval over the bandwidth varying network, since edges of different structures are often the most important features for recognizing the image, it is usually desirable to display or recognize the shape of an image at very low bit rates, and then progressively enhance it as more bits are received. Various progressive or scalable wavelet image coding algorithms based on edges and surfaces of an image were proposed $^{5-7}$.

In hierarchical octave-band wavelet image coding, traditional scalable coding techniques encode an image starting from the lowest subband. At very low bit rate, the low frequency information together with the blurred and blotchy artifacts will dominate the decoded image. To enhance the visual appearance and recognizability of the decoded image at very low bit rates, a progressive coding scheme based on edge information was suggested ${ }^{6}$. In ref. 6 , the high resolution subbands is encoded first. This is because the visually important features, such as the skeleton, can be encoded, decoded and displayed first by encoding wavelet coefficients in these high resolution subbands. Although the bit rate can be reduced without any significant degradation on the decoded image quality, numerous bits are still needed to encode the simplest edges that make the coding algorithm inefficient.

Another edge-enhanced image coding algorithm was proposed in ${ }^{5}$. In ref. 5, edge detection is integrated into the SPIHT algorithm ${ }^{3}$. The detected edges are encoded, transmitted and decoded separately, and then combined with decoded images at very low bit rates to produce edge-enhanced images. Although this approach allows the recognition of the shape of an image at very low bit rates, and the edge detection algorithm SUSAN is insensitive to noise and has good edge localization, the involved multi-ring chain coding scheme, which is effective for long and smooth curves only, is too costly for most images, especially for those contain many fine textures or patterns. Moreover, the method for the combination of the decoded edges and decoded images is also very complicated.

According to the properties of the human visual system (HVS), surface orientation is also important for the recognition and the extraction of visual objects. Therefore, another scalable coding algorithm based on the surface orientation was proposed ${ }^{7}$. It was suggested that the gray levels should be maintained at all time in order to have a 'good impression' of the decoded images. The surface orientation determined from the shadings is used as the criteria to decide the order of the subbands and their bit-planes for encoding and transmission. The surface orientation is measured by the stereographic projection of the reflectance map obtained from the image brightness. With this algorithm, coarse shape of objects is first decoded, with shading information added progressively. This avoids using many bits for edge coding, like the above edgeenhanced algorithms. However, since the mathematical relation between the wavelet coefficients at different scales and orientations, and the surface orientation is complicated and still not concrete, only a trial method can be employed. Moreover, due to the assumption of point light source and Lambertian object surfaces, the scope of images suitable for this algorithm is limited. Furthermore, since the decoded images at high bit rate is closed to the original one, it becomes very difficult to determine the transmission sequence as the surface orientations of the several consecutively decoded 
images are similar. With these unsolvable problems, it still cannot be considered as a desirable feature-based scalable wavelet image coding algorithm.

\section{Estimation of the Lipschitz Regularity}

\subsection{Definitions}

Denote $\mathbf{x}=\left(x_{1}, x_{2}\right), \mathbf{u}=\left(u_{1}, u_{2}\right)$ and $\mathbf{v}=\left(v_{1}, v_{2}\right)$. Let $p_{\mathbf{v}}(\mathbf{x})$ be a local polynomial in the neighborhood of $\mathbf{v}$ and $\left(\mathrm{D}_{i}\right)^{r}=\frac{\partial^{r}}{\partial x_{i}^{r}}$ be a differential operator such that

$$
p_{\mathbf{v}}(\mathbf{x})=\sum_{k=0}^{m-1} \frac{\left[\left(\left(x_{1}-v_{1}\right) \mathrm{D}_{1}+\left(x_{2}-v_{2}\right) \mathrm{D}_{2}\right)^{k} f\right](\mathbf{v})}{k !} \text {. }
$$

If $f$ is in $C^{m}$, that is $f$ is $m$ times continuously differentiable in the neighborhood of $\mathbf{v}$, where $\mathbf{v} \in \mathfrak{R}^{2}$, then over a small interval, $f$ can be well approximated by $p_{\mathbf{v}}(\mathbf{x})$, that is

$$
f(\mathbf{x})=p_{\mathbf{v}}(\mathbf{x})+\varepsilon_{\mathbf{v}}(\mathbf{x})
$$

where $\varepsilon_{\mathbf{v}}(\mathbf{x})$ is the approximation error in the neighborhood of $\mathbf{x}$, and

$$
\left|\varepsilon_{\mathbf{v}}(\mathbf{x})\right| \leq \frac{\sup _{\mathbf{u} \in \mathbf{U}(\mathbf{v})}\left[\left(\left(x_{1}-v_{1}\right) \mathrm{D}_{1}+\left(x_{2}-v_{2}\right) \mathrm{D}_{2}\right)^{m} f\right](\mathbf{u})}{m !},
$$

where $\mathrm{U}(\mathbf{v})$ denotes the neighborhood of $\mathbf{v}$.

Definition 1: A function $f \in L^{2}\left(\Re^{2}\right)$ is called a pointwise Lipschitz with $\alpha \geq 0$ at $\mathbf{v}$, if there exists $K>0$, and a polynomial $p_{\mathbf{v}}$ of degree $m=\lfloor\alpha\rfloor$ such that $\forall \mathbf{x}, \mathbf{v} \in \mathfrak{R}^{2}$,

$$
\left|f\left(x_{1}, x_{2}\right)-p_{\mathbf{v}}\left(x_{1}, x_{2}\right)\right| \leq K\left(\left|x_{1}-v_{1}\right|^{2}+\left|x_{2}-v_{2}\right|^{2}\right)^{\frac{\alpha}{2}} .
$$

If $f$ is $m=\lfloor\alpha\rfloor$ times continuously differentiable in $\mathrm{U}(\mathbf{v})$, then $p_{\mathrm{v}}$ is the Taylor expansion of $f$ at $\mathbf{v}$ and the polynomial $p_{\mathbf{v}}(\mathbf{x})$ is uniquely defined for any $\mathbf{v}$. Since pointwise Lipschitz exponents may vary arbitrary from abscissa to abscissa, to obtain a more global measurement of regularity over a region $\mathrm{U}(\mathbf{v})$, we need to define uniform Lipschitz regularity. 
Definition 2: Let $0 \leq \alpha \leq 1$. A function $f \in L^{2}\left(\Re^{2}\right)$ is called a uniformly Lipschitz with $\alpha$ in $\mathrm{U}(\mathbf{v})$, if there exists $K>0$ which is independent of $\mathbf{v}$, and a polynomial $p_{\mathbf{v}}$ of degree $m=\lfloor\alpha\rfloor$ such that $\forall \mathbf{v} \in \mathrm{U}(\mathbf{v}), \forall \mathbf{x} \in \Re^{2}$,

$$
\left|f\left(x_{1}, x_{2}\right)-f\left(v_{1}, v_{2}\right)\right| \leq K\left(\left|x_{1}-v_{1}\right|^{2}+\left|x_{2}-v_{2}\right|^{2}\right)^{\frac{\alpha}{2}} .
$$

If the Lipschitz exponent is smaller than 1 , then $f$ is not differentiable at $\mathbf{v}$ and $\alpha$ characterizes the singularity type.

\subsection{Vanishing moments and decay of the wavelets}

Since the vanishing moment of the mother wavelet has an important role in characterizing the regularity of an image, we denote $\psi^{l}(\mathbf{x})$ as mother wavelets with $n$ vanishing moments for $1 \leq l \leq 3$, where $l$ is the index of orientation and it is discussed in Section 3.3. A wavelet $\psi^{l}(\mathbf{x})$ having $n$ vanishing moments is orthogonal to polynomials of degree $n-1$. When we estimate the Lipschitz exponents of $f$, we often ignore the wavelet transform of $p_{\mathrm{v}}$ in Eq. (1) if a wavelet $\psi^{l}(\mathbf{x})$ has $n>\lfloor\alpha\rfloor$ vanishing moments. It is because if $n>\lfloor\alpha\rfloor$, then $p_{\mathrm{v}}$ has degree of at most $n-1$. With the change of variable, the wavelet transform of $p_{\mathrm{v}}$ is

$$
\mathrm{W}_{j} p_{\mathbf{v}}(\mathbf{u})=0 .
$$

Suppose that the wavelet $\psi^{l}(\mathbf{x})$ has $n$ vanishing moments and is $n$ times continuously differentiable with fast decay, that is for any natural number $q$ and any $0 \leq k \leq n$, there exists a constant $C_{q}$ such that $\forall \mathbf{x} \in \mathfrak{R}^{2}$,

$$
\left|\nabla^{k} \psi^{l}(\mathbf{x})\right| \leq \frac{C_{q}}{\left(1+x_{1}^{q}\right)\left(1+x_{2}^{q}\right)}
$$

If $f$ has $n$ vanishing moments, then the wavelet transform of $f$ can be interpreted as a multiscale differential operator of order $n$. Hence, there is a relationship between the differentiability of $f$ and the decay of the wavelet transform at fine scales.

\subsection{Definition of the separable wavelet transform}

Recall that $l$ is the index of orientation and $\mathbf{x}=\left(x_{1}, x_{2}\right), \mathbf{u}=\left(u_{1}, u_{2}\right)$. Let $\varphi$ be a scaling function such that 


$$
\int_{-\infty}^{+\infty} \varphi(\mathbf{x}) \mathrm{d} \mathbf{x}=1
$$

and $\psi$ be the corresponding wavelet that generates a wavelet orthonormal basis of $L^{2}\left(\Re^{2}\right)$. The set of separable mother wavelets $\left\{\psi^{l}\right\}_{1 \leq 1 \leq 3}$ can then be constructed with tensor products of the scaling function $\varphi$ and the wavelet function $\psi$. That is, $\psi^{1}(\mathbf{x})=\varphi\left(x_{1}\right) \psi\left(x_{2}\right), \psi^{2}(\mathbf{x})=\psi\left(x_{1}\right) \varphi\left(x_{2}\right)$ and $\psi^{3}(\mathbf{x})=\psi\left(x_{1}\right) \psi\left(x_{2}\right)$. Let $\psi_{j, \mathbf{u}}^{l}(\mathbf{x})$ be the set of translations and dilations of the mother wavelets $\left\{\psi^{\prime}\right\}_{1 \leq 1 \leq 3}$, that is $\psi_{j, \mathbf{u}}^{l}(\mathbf{x})=2^{-j} \psi^{l}\left(2^{-j} x_{1}-u_{1}, 2^{-j} x_{2}-u_{2}\right)$, then $\left\{\psi_{j, \mathbf{u}}^{l}\right\}_{1 \leq 1 \leq 3}$ is an orthonormal basis of $L^{2}\left(\Re^{2}\right)$ as shown in Figure 1. Let $\varphi_{J, \mathrm{u}}^{2}(\mathbf{x})=2^{-J} \varphi^{2}\left(2^{-J} x_{1}-u_{1}, 2^{-J} x_{2}-u_{2}\right)$, where $\varphi^{2}(\mathbf{x})=\varphi\left(x_{1}\right) \varphi\left(x_{2}\right)$ and $J$ is the total number of scales. Then the separable wavelet transform of a signal $f \in L^{2}\left(\Re^{2}\right)$ at $\mathbf{u}$ is defined as:

$$
\left\{S_{J} f(\mathbf{u})=\left\langle f, \varphi_{J, \mathbf{u}}^{2}\right\rangle,\left(\mathrm{W}_{j}^{l} f(\mathbf{u})=\left\langle f, \psi_{j, \mathbf{u}}^{l}\right\rangle\right)_{1 \leq j \leq J, 1 \leq 1 \leq 3}\right\},
$$

where $S_{J} f$ and $\mathrm{W}_{j}^{l} f$ represent the scaling coefficients and the wavelet coefficients respectively.

The three mother wavelets can be used to extract image details at different scale. So wavelet coefficients at different scales and orientations can fully represent different features of an image.

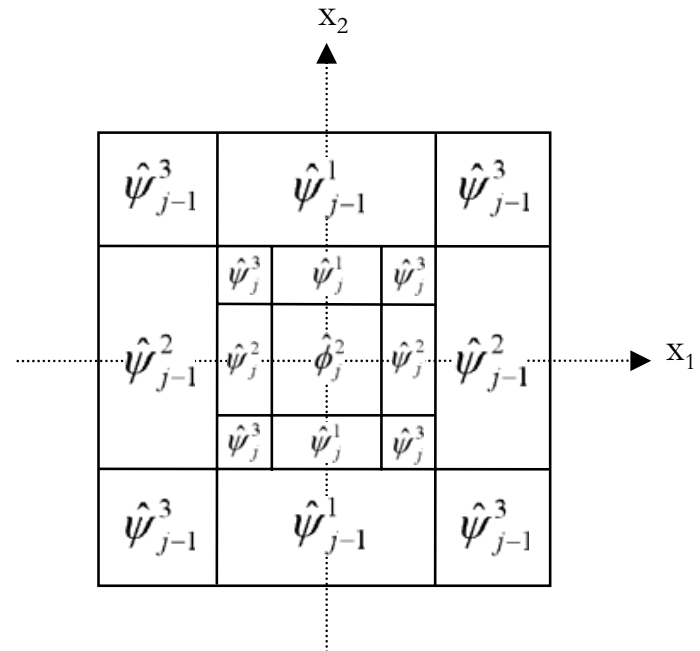

Fig. 1. Dyadic rectangles indicating the frequency regions for which the energy of $\psi_{j, \mathbf{u}}^{l}(\mathbf{x})$ is mostly concentrated. 


\subsection{Uniform Lipschitz regularity for the separable dyadic wavelet transform}

Let $\mathrm{U}_{\delta}(\mathbf{v})=\left\{\mathbf{u}:\left|u_{1}-v_{1}\right| \leq \varepsilon+\delta,\left|u_{2}-v_{2}\right| \leq \varepsilon+\delta\right\}$ for $\delta>0$ be the region that belongs to the support of $\psi_{j, \mathbf{u}}^{l}(\mathbf{x})$, that is the cone of influence which is denoted by $\operatorname{COI}_{j}^{l}(\mathbf{v})$ for $1 \leq j \leq J$ and $1 \leq l \leq 3$, where $J$ is the total number of scales. Let $n$ be the order of the multiscale differential operator of $f$, then we have the following theorem.

Theorem 1: If $f$ is uniformly Lipschitz with $\alpha \leq n$ in the neighborhood of $\mathbf{v}$ defined by $U_{\delta}(\mathbf{v})$, then there exists a finite constant $A>0$ such that

$$
\forall(j, \mathbf{u}) \in S^{(j, \mathbf{v})},\left|\mathrm{W}_{j}^{l} f(\mathbf{u})\right| \leq A\left(2^{j}\right)^{\alpha}
$$

Conversely, suppose that $f$ is bounded and $\alpha<n$ is not an integer. If there exists a finite constant $A^{\prime}>0$ and $\alpha^{\prime}<\alpha$ such that

$$
\forall(j, \mathbf{u}) \in S^{(j, \mathbf{v})},\left|\mathrm{W}_{j}^{l} f(\mathbf{u})\right| \leq A^{\prime}\left(2^{j}\right)^{\alpha^{\prime}}
$$

then $f$ is Lipschitz with $\alpha$ in $U_{\delta}(\mathbf{v})$.

Proof: The proofs of Eq. (8) and Eq. (9) are shown in Appendix A and B, respectively.

\subsection{Lipschitz regularity estimation from the decimated separable wavelet transform}

By theorem 1, it is plausible to characterize the regularity from the evolution of the coefficients generated from the decimated separable wavelet transform. Experimental observation indicates that strong edges achieve equality in Eq. $(8)^{14}$. This is the extreme case. In general, different levels of Lipshitz regularity can be distinguished and obtained by computing the interscale ratios of the decimated wavelet coefficient magnitudes. Wavelet zero-tree coding algorithm also takes the advantage of the decay of decimated wavelet coefficients by exploiting the relationship between the wavelet coefficients at different scales and orientations and their quad-trees. Here, quad-trees refer to the four children of each wavelet coefficient $\mathrm{W}_{j}^{l} f[\mathbf{u}]$ to the next fine scale $2^{j-1}$ , that is $\quad \mathrm{W}_{j-1}^{l} f\left[2 u_{1}, 2 u_{2}\right], \quad \mathrm{W}_{j-1}^{l} f\left[2 u_{1}-1,2 u_{2}\right], \quad \mathrm{W}_{j-1}^{l} f\left[2 u_{1}, 2 u_{2}-1\right] \quad$ and $\mathrm{W}_{j-1}^{l} f\left[2 u_{1}-1,2 u_{2}-1\right]$. At the largest scale $2^{J}$, the children of $\mathrm{S}_{J} f[\mathbf{u}]$ are defined as the three wavelet coefficients at the same scale and location $\mathrm{W}_{J}^{1} f[\mathbf{u}], \mathrm{W}_{J}^{2} f[\mathbf{u}]$ and $\mathrm{W}_{J}^{3} f[\mathbf{u}]$. The construction of the quad-trees is illustrated in Figure 2. The values of a wavelet coefficient and its four children depend on the gray level variation of the image in the same spatial area. So we compute the interscale ratios as the following, 


$$
\mathrm{R}_{j-1}^{l} f[\mathbf{u}]=\frac{\left|\mathrm{W}_{j}^{l} f[\mathbf{u}]\right|}{\sum_{u_{1}^{\prime}=0}^{1} \sum_{u_{2}^{\prime}=0}^{1}\left|\mathrm{~W}_{j-1}^{l} f\left[2 u_{1}-u_{1}^{\prime}, 2 u_{2}-u_{2}^{\prime}\right]\right|}
$$

and at the coarse scale $j=J$,

$$
\mathrm{R}_{J}^{l}[\mathbf{u}]=\frac{\mid \mathrm{S}_{J} f[\mathbf{u}]}{\left|\mathrm{W}_{J}^{l} f[\mathbf{u}]\right|}
$$

In our adaptive scalable coding algorithm, different levels of regularity of the decimated separable wavelet transformed image are estimated by the following equation:

$$
\mathrm{R}_{j-1}^{l}[\mathbf{u}]=2^{\alpha+2} .
$$

Apart from this, finer estimation of regularity can also be performed with the expense of computation by computing the interscale ratios for each coefficient at the fine scales, that is:

$$
\mathrm{R}_{j-1}^{l}\left[2 u_{1}-u_{1}^{\prime}, 2 u_{2}-u_{2}^{\prime}\right]=\frac{\left|\mathrm{W}_{j}^{l} f[\mathbf{u}]\right|}{\left|\mathrm{W}_{j-1}^{l} f\left[2 u_{1}-u_{1}^{\prime}, 2 u_{2}-u_{2}^{\prime}\right]\right|} \text { for } u_{1}^{\prime}, u_{2}^{\prime}=0,1,
$$

and

$$
\mathrm{R}_{j-1}^{l}[\mathbf{u}]=2^{\alpha}
$$

Therefore, the children belonging to a parent can be set to zeros when they are found to be above a certain level of regularity. 
10 C. Y. F. Ho, T. C. Hsung, D. P. K. Lun, B. W. K. Ling, P. K. S. Tam and W. C. Siu
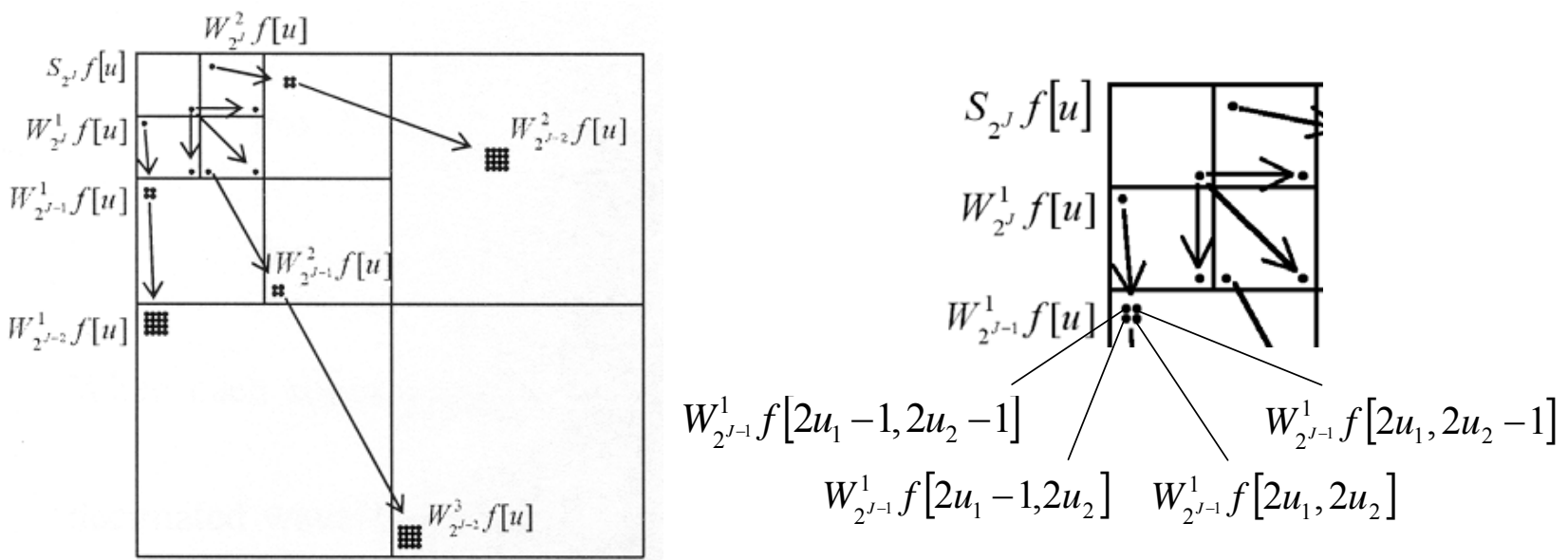

Fig. 2. The decimated separable wavelet transform and the construction of quad-trees.

\subsection{Decimated separable wavelet transform magnitude sum over the 'cone of influence'}

It has been shown in ref. 13 that the interscale ratios of the magnitude sums over the 'cone of influence' can provide a fast and effective estimation of Lipschitz regularity. Before determining the 'cone of influence', that is the regions for the magnitude sums, for the decimated case, let us consider the undecimated case first. Suppose $\varphi$ and $\psi$ have compact supports $\left[-K_{1}, K_{1}\right]$ and $\left[-K_{2}, K_{2}\right]$, respectively. Denote $\mathbf{n}=\left(n_{1}, n_{2}\right)$. The regions for the magnitude sums at position $\mathbf{u}$, scale $j$ and orientation $l$ over the undecimated separable wavelet transform are

$$
\begin{aligned}
& \operatorname{COI}_{j}^{1}[\mathbf{u}]=\left\{\mathbf{n}:\left|n_{1}-u_{1}\right| \leq K_{1} 2^{j},\left|n_{2}-u_{2}\right| \leq K_{2} 2^{j}\right\}, \\
& \operatorname{COI}_{j}^{2}[\mathbf{u}]=\left\{\mathbf{n}:\left|n_{1}-u_{1}\right| \leq K_{2} 2^{j},\left|n_{2}-u_{2}\right| \leq K_{1} 2^{j}\right\}, \\
& \operatorname{COI}_{j}^{3}[\mathbf{u}]=\left\{\mathbf{n}:\left|n_{1}-u_{1}\right| \leq K_{2} 2^{j},\left|n_{2}-u_{2}\right| \leq K_{2} 2^{j}\right\},
\end{aligned}
$$

and

$$
\operatorname{COI}_{J}^{4}[\mathbf{u}]=\left\{\mathbf{n}:\left|n_{1}-u_{1}\right| \leq K_{1} 2^{J},\left|n_{2}-u_{2}\right| \leq K_{1} 2^{J}\right\}
$$

When each subband is downsampled by $2^{j}$, the positions are shifted and scaled as shown in Figure 3. So we define the 'cone of influence' for the decimated separable wavelet transform as follow,

$$
\operatorname{dCOI}_{j}^{1}[\mathbf{u}]=\left\{\mathbf{n}:\left|\left(n_{1}-u_{1}\right) / 2^{j}\right| \leq K_{1},\left|\left(n_{2}-u_{2}\right) / 2^{j}\right| \leq K_{2}\right\},
$$




$$
\begin{aligned}
& \operatorname{dCOI}_{j}^{2}[\mathbf{u}]=\left\{\mathbf{n}:\left|\left(n_{1}-u_{1}\right) / 2^{j}\right| \leq K_{2},\left|\left(n_{2}-u_{2}\right) / 2^{j}\right| \leq K_{1}\right\}, \\
& \operatorname{dCOI}_{j}^{3}[\mathbf{u}]=\left\{\mathbf{n}:\left|\left(n_{1}-u_{1}\right) / 2^{j}\right| \leq K_{2},\left|\left(n_{2}-u_{2}\right) / 2^{j}\right| \leq K_{2}\right\},
\end{aligned}
$$

and

$$
\mathrm{dCOI}_{J}^{4}[\mathbf{u}]=\left\{\mathbf{n}:\left|\left(n_{1}-u_{1}\right) / 2^{J}\right| \leq K_{1},\left|\left(n_{2}-u_{2}\right) / 2^{J}\right| \leq K_{1}\right\}
$$

We then compute the magnitude sums as follows:

$$
\mathrm{N}_{j}^{l}[\mathbf{u}]=\sum_{\mathbf{n} \in \mathrm{dCOI}_{j}^{l}[\mathbf{u}]}\left|\mathrm{W}_{j}^{l} f[\mathbf{n}]\right|,
$$

and

$$
\mathrm{N}_{J}[\mathbf{u}]=\sum_{\mathbf{n} \in d \mathrm{dCOI}_{J}^{+}[\mathbf{u}]}\left|\mathrm{S}_{f} f[\mathbf{n}]\right|
$$

Similar to Eq. (10) and Eq. (12), we obtain the interscale ratios as,

$$
\mathrm{R}_{j-1}^{l}[\mathbf{u}]=\frac{\mathrm{N}_{j}^{l}[\mathbf{u}]}{\sum_{u_{1}^{\prime}=0 u_{2}^{\prime}=0}^{1} \sum_{j-1}^{1} \mathrm{~N}_{j}^{l}\left[2 u_{1}-u_{1}^{\prime}, 2 u_{2}-u_{2}^{\prime}\right]},
$$

or

$$
\mathrm{R}_{j-1}^{l}\left[2 u_{1}-u_{1}^{\prime}, 2 u_{2}-u_{2}^{\prime}\right]=\frac{\mathrm{N}_{j}^{l}[\mathbf{u}]}{\mathrm{N}_{j-1}^{l}\left[2 u_{1}-u_{1}^{\prime}, 2 u_{2}-u_{2}^{\prime}\right]} \text { for } u_{1}^{\prime}, u_{2}^{\prime}=0,1 \text {, }
$$

and at the coarsest scale $j=J$,

$$
\mathrm{R}_{J}^{l}[\mathbf{u}]=\frac{\mathrm{N}_{J}[\mathbf{u}]}{\mathrm{N}_{J}^{l}[\mathbf{u}]}
$$

Similar to the result from ref. 13 and Eq. (8), we have

$$
\mathrm{N}_{j}^{l}[\mathbf{u}] \leq A^{\prime \prime}\left(2^{j}\right)^{\alpha+1},
$$

where $A^{\prime \prime}>0$ is a finite constant. From Eq. (11) and Eq. (13), we also have the interscale ratios for the magnitude sum over the 'cone of influence' as

$$
\mathrm{R}_{j}^{l}[\mathbf{u}]=2^{\alpha+3},
$$


or

$$
\mathrm{R}_{j}^{l}[\mathbf{u}]=2^{\alpha+1} .
$$

As a result, we avoid the complicated procedure from the direct computation of the Lipschitz exponents. Hence, the errors and ambiguities that occur when tracing the evolution of the wavelet transform coefficient magnitudes at coarse scales are avoided.

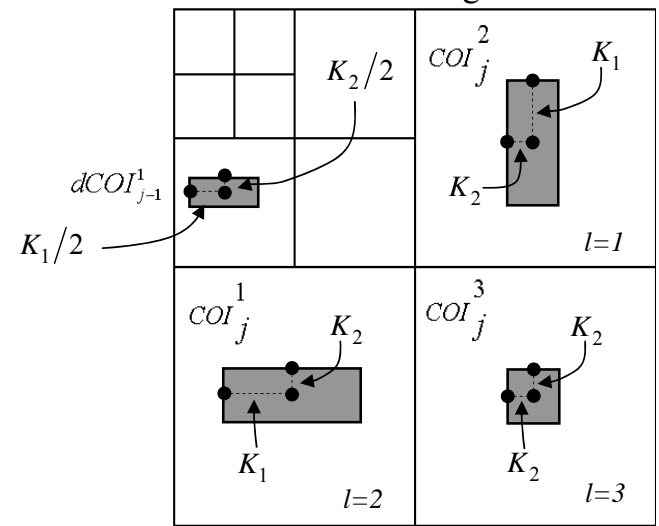

Fig. 3. The decimated COI of the separable discrete wavelet transform.

\section{Our Proposed Algorithm}

The technique which employs regularity as a post-processing to preserve the edges of the noisy natural or tomographic images was proposed in ref. 13. Although there are some common points between the work in ref. 13 and that in this paper, such as the estimation of the regularity of the signals or images via the WTMS approach, there are lots of differences. The work in ref. 13 was for denoising applications, but not for coding applications. The difference between these two applications is that the size of data is not important for denoising applications, while it is extremely critical for coding applications. Hence, all processing for denoising applications can be performed in the undecimated domain. However, all processing for the coding applications are needed to perform in the decimated domain. One of the contributions of this paper is to show that the technique for the estimation of the regularity of a signal can really be applied in the decimated domain. Besides, denoising applications emphasize on the determination of the threshold, which is determined based on the regularity of the signal ${ }^{13}$, so that the SNR is improved. On the other hand, coding applications emphasize on the selection of the appropriate coefficients, which is also based on the regularity of the signal, and the combining of these selected coefficients in a sequence for encoding and transmission, so that the coding gain is maximized. Since the objectives of these two applications are completely different, the ways to employ the regularity property are also completely different.

Since we have shown in the previous section that we can estimate the Lipschitz regularity from the interscale ratios of the transformed coefficient magnitudes or the magnitude sums over the 'cone of influence', in this section, we will focus on the 
methods for the selection of the appropriate coefficients based on the regularity of an image and combining these selected coefficients in a sequence.

The proposed algorithm is shown as follows.

\section{Procedures:}

Step 1: Obtain the decimated wavelet coefficients as Eq. (7) from the existing wavelet image encoder.

Step 2: Compute the magnitude sums using Eq. (14), where $1 \leq j \leq J$ and $1 \leq l \leq 3$.

Step 3: Compute the interscale ratios using Eq. (15a) and Eq. (15b).

Step 4: Normalize the magnitudes of the interscale ratios individually before combining them.

Step 5: Sort these magnitudes of the interscale ratios in ascending order.

Step 6: Determine $\alpha_{i}$ for $i=1,2, \ldots, L$, where $L$ is the total number of regularity levels.

Step 7: Set $\mathrm{R}_{j, i}^{l}[\mathbf{u}]=2^{\alpha_{i}+1}$ for each $i=1,2, \ldots, L$.

Step 8: Starting at $j=J$, for regularity level $i$, select $\mathrm{W}_{j}^{l} f[\mathbf{u}]$ if $\mathrm{R}_{j}^{l} f[\mathbf{u}] \leq \mathrm{R}_{j, i}^{l} f[\mathbf{u}]$ $\forall j, l$.

In Step 1, we obtain the wavelet coefficients from the image coder. Traditionally, a jointly shiftable wavelet transform ${ }^{15}$ with the wavelet filters having compact supports in time domain is used to represent multiscale signals and perform image analysis because the transform coefficients are translation invariant in position and orientation. There is also no aliasing effect upon subsamplings and zero interpolation. However, since our objective is to compress images, it is more desirable to obtain a representation of signal information with a minimal degree of correlation and redundancy but a maximal concentration of energy distribution, so that the coding gain is maximized. Therefore, regularity estimation is conducted via the biorthogonal wavelet filters employed in the existing image coder. Moreover, using these biorthogonal wavelet filters can be compatible to the existing wavelet image coder. The interscale ratios of the magnitude sums defined in Steps 2 and 3 can be obtained easily. Since wavelet coefficients will have large amplitudes when the signal has sharp transitions, the important visual information are found in the wavelet coefficients with large amplitude. This property can be captured into the wavelet image coder via normalizing the interscale ratios by the magnitude of the wavelet coefficients, which is described in Step 4 of the procedures, that the interscale ratios of the magnitude sums are normalized by a larger factor if the corresponding wavelet coefficients have larger magnitude. Moreover, since the dynamic ranges of different images are different, normalization on these interscale ratios to the same range are required before 
combining them via a simple matrix multiplication. Then the combined values are sorted in Step 5 and the coefficients are selected using the criteria defined in Steps 7 and 8 so that those coefficients with larger magnitudes and smaller interscale ratios have a higher priority to be selected and encoded. The regularity levels defined in Step 6 are in an even progression. This arrangement of the bitstream can effectively increase the coding efficiency of the regularity scalable coder and the result will be shown in Section 5.

Since the regularity levels are determined adaptively from the images, a full range of regularity levels can be utilized and better image quality can be achieved. Figure 4 shows how this proposed scalable coding algorithm (Steps 7 and 8) is embedded in the zero-tree wavelet image encoder. Layers of selected wavelet coefficients at different regularity levels are subsequently quantized and entropy coding is performed to form a regularity and resolution scalable bitstream for transmission. Note that the level of regularity of the reconstructed image depends on the bit rate budget, the required resolution and the employed quantization steps.

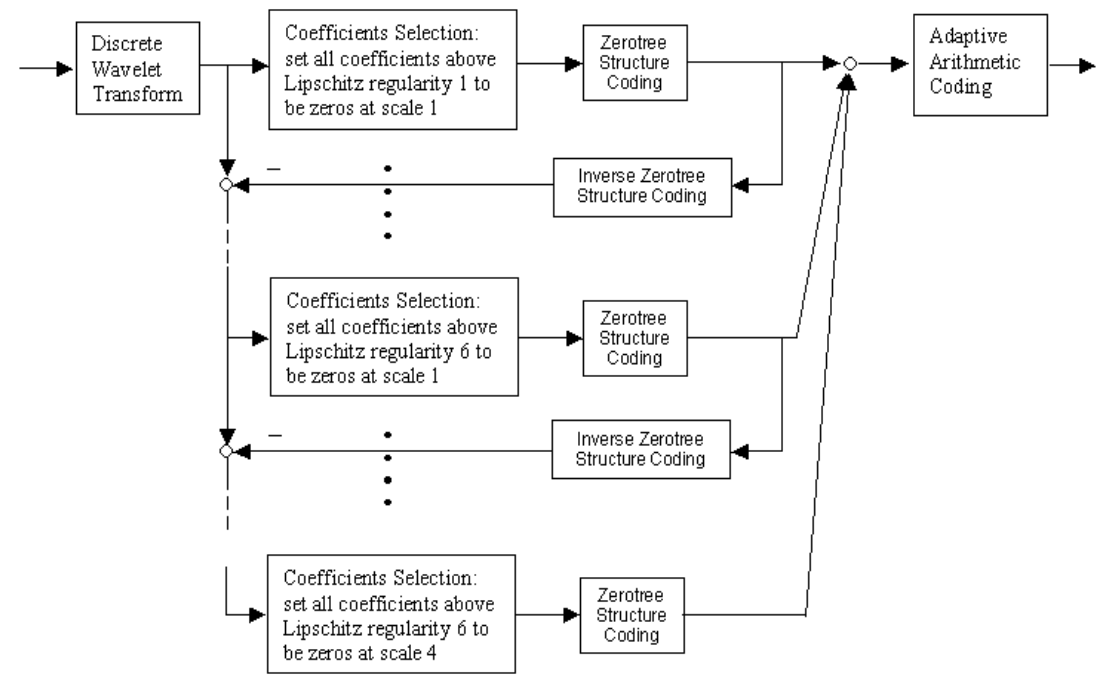

Fig. 4. Embedding the proposed regularity scalable coding algorithm into the existing wavelet zero-tree image encoder.

\section{Simulation Results}

We adopt the proposed algorithm to the MPEG-4 still image codec with the popular Daubechies $9 / 3$ biorthogonal filters $\left(K_{1}=4, K_{2}=1\right)$ for an illustration. In fact, our proposed regularity scalable coder can work with any kind of scalable wavelet coding techniques, such as the JPEG2000 coding scheme, without changing the bitstream format. However, we choose the MPEG-4 still image codec for an illustration because images are broken down into several pieces in the JPEG2000 coding scheme and wavelets transforms are applied on each pieces. As a result, the number of interscales of wavelet coefficients in the JPEG2000 coding scheme is usually less than that of the 
MPEG-4 still image codec. For our proposed regularity scalability coding scheme, since one of the objectives and important advantages of our proposed approach is to introduce regularity scalability in addition to the resolution scalability without a change in the bitstream format, more interscales of wavelet coefficients would provide more scalable levels. Hence, we choose the MPEG-4 still image codec for an illustration.

To compare the quantitative performance of our proposed coder with that of the existing coder, the PSNR against the bit rate at each resolution scale is shown in Figure 5. The overall PSNR for all resolution scales of our scalable coding algorithm outperforms significantly than that of the original coding algorithm with resolution scalability only at the similar bit rates. This implies that if the original image coder can compress images at a certain bit rate, our proposed regularity scalable coder can also compress the same images with similar bit rates, but higher PSNR.

To compare the qualitative performance of our proposed coder with that of the existing coder, the well-known USC image "Lena" are presented for the comparison. Table 1 shows the quantitative results of both our proposed coding algorithm and the existing image coding algorithm (Comparison A in Figure 5a). Figures 6 and 7 show the qualitative results. At resolution scale 2, when the quantization step size is equal to 6 , we can see that up to $34.98 \%$ reduction in bit rate can be obtained without significant change in the visual quality ( $3{ }^{\text {rd }}$ row of Figure 6 ) and only less than $2.53 \%$ reduction in PSNR is resulted for encoding the image "Lena". That means the low regularity components, such as the edges and textures, are enhanced significantly with a small addition of the bit rate. The significant improvement can also be noted in the Comparison B in Figure 5a. The bit rate of the image can be enormously reduced from $1.4898 \mathrm{bpp}$ to $0.58475 \mathrm{bpp}$ (a reduction of $60.75 \%$ ) without markedly change in PSNR (a reduction of $3.08 \%$ ). The results of the visual quality of the images in the Comparison A shown in Figure 6 show the success of the proposed algorithm.

Zooming into different parts of the decoded image as shown in Figure 7, we assure that there is almost no degradation of the visually important features, such as the edges and the hat strings, upon a significant reduction in the bit rate. This is because with the proposed algorithm, these low regularity components suffer less to the distortion. Severe degradation just exists in the smooth areas, such as the shoulder, but it is not visually annoying as it just gives a smoothening effect. Since the order of the embedded coding begins at the largest quantization step which is progressively decreasing, the coding precision increases as the number of quantization refinements increases. Therefore, to visualize the qualitative and quantitative improvements of the regularity scalability, we preserve the highest precision without performing quantization, as the results shown in Figures 5 and 7.

Recall that ref. 5 suggests encoding the higher resolution subbands first, so that the visually important edges are decoded and displayed first. On the other hand, it is traditionally suggested that the lowest resolution subband should be encoded first. Here we adopt our proposed algorithm into these two transmission schemes. Results of the well-known USC images "Pepper" are presented in Figure 8. For the transmission scheme based on ref. 5, the result is shown in Figure 8a. It is worth noting that the overall quality of the reconstructed images under our proposed algorithm outperforms than that of ref. 7 and the quality of the image is enhanced significantly at very low bit rates. For the comparison with the results in ref. 7, which is shown in Figure 8b, we also note that the overall quality of the reconstructed image using our proposed 
approach outperforms than that of ref. 5. In particularly, the boundary shapes and edges of the decoded images are improved significantly.

To compare the complexity of the proposed coder, since it only involves the computation of magnitude sums and interscale ratios in the decimated domain, the computational cost is low. For the existing edge-enhanced scalable coding techniques ${ }^{5-}$ ${ }^{6}$, edge detection is required and these algorithms are performed in the undecimated domain. Obviously, the computational cost of these algorithms is much higher than that of our proposed algorithm.

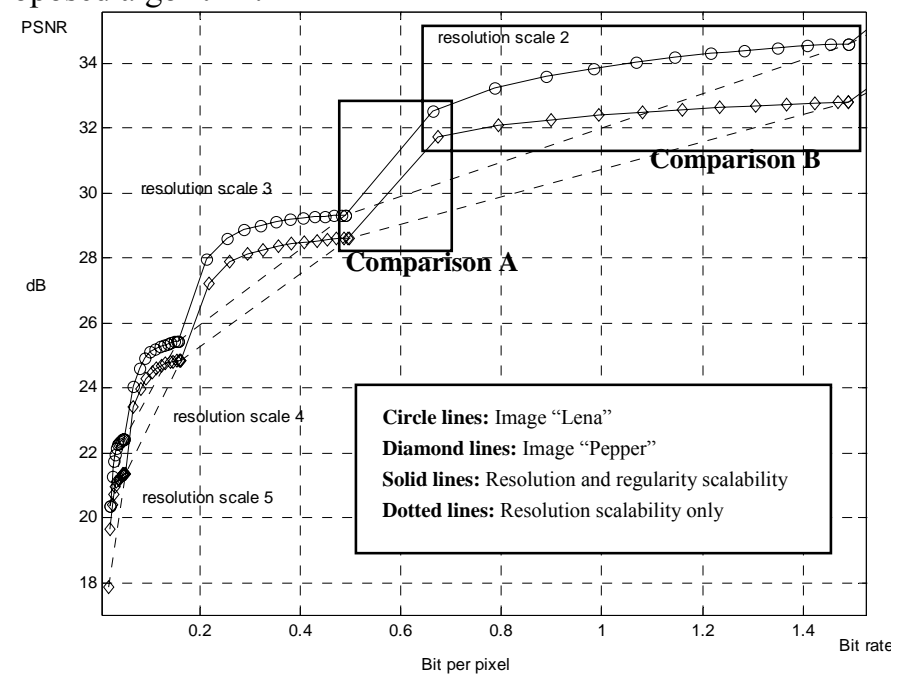

(a)

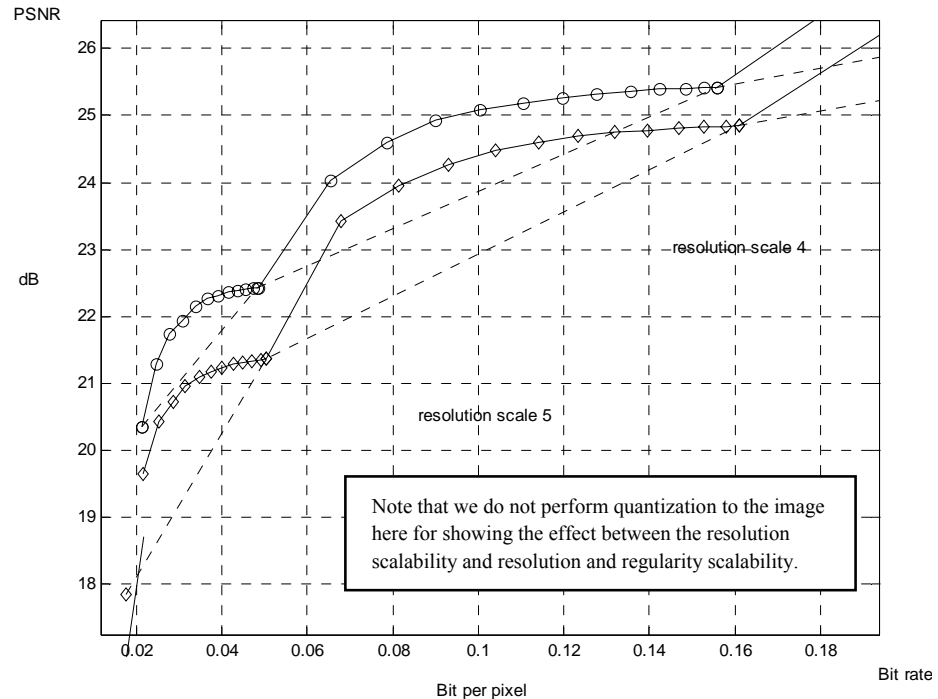

(b)

Fig. 5. Performances of resolution scalability versus resolution and regularity scalability at resolution scales (a) 2-5. (b) 4 and 5 . 
Table 1. Effect of quantization to the regularity scalable coding at resolution scale 2.

\begin{tabular}{|c|c|c|c|c|c|c|}
\hline & \multicolumn{2}{|c|}{ Resolution scalable } & \multicolumn{2}{c|}{ Regularity and resolution scalable } & \multicolumn{2}{c|}{ Percentage change } \\
\hline $\begin{array}{c}\text { Quantization } \\
\text { step size }\end{array}$ & PSNR (dB) & Bit rates (bpp) & PSNR (dB) & Bit rates (bpp) & PSNR (\%) & Bit rates (\%) \\
\hline 2 & 34.5795 & 1.49017 & 33.5911 & 0.83072 & -2.86 & -44.25 \\
\hline 4 & 34.5229 & 1.14035 & 33.5762 & 0.67340 & -2.74 & -40.95 \\
\hline 6 & 34.4211 & 0.89929 & 33.5495 & 0.58475 & -2.53 & -34.98 \\
\hline 8 & 34.3012 & 0.73837 & 33.5113 & 0.52167 & -2.30 & -29.35 \\
\hline 32 & 32.5595 & 0.25671 & 32.4216 & 0.24667 & -0.42 & -3.91 \\
\hline 64 & 30.4063 & 0.13934 & 30.4040 & 0.13931 & -0.01 & -0.02 \\
\hline
\end{tabular}


18 C. Y. F. Ho, T. C. Hsung, D. P. K. Lun, B. W. K. Ling, P. K. S. Tam and W. C. Siu
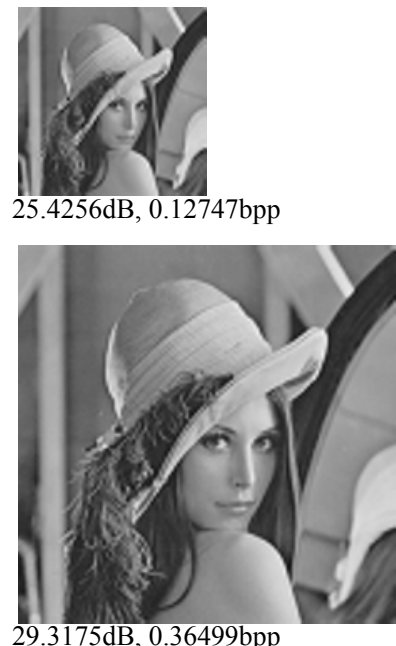

$29.3175 \mathrm{~dB}, 0.36499 \mathrm{bpp}$

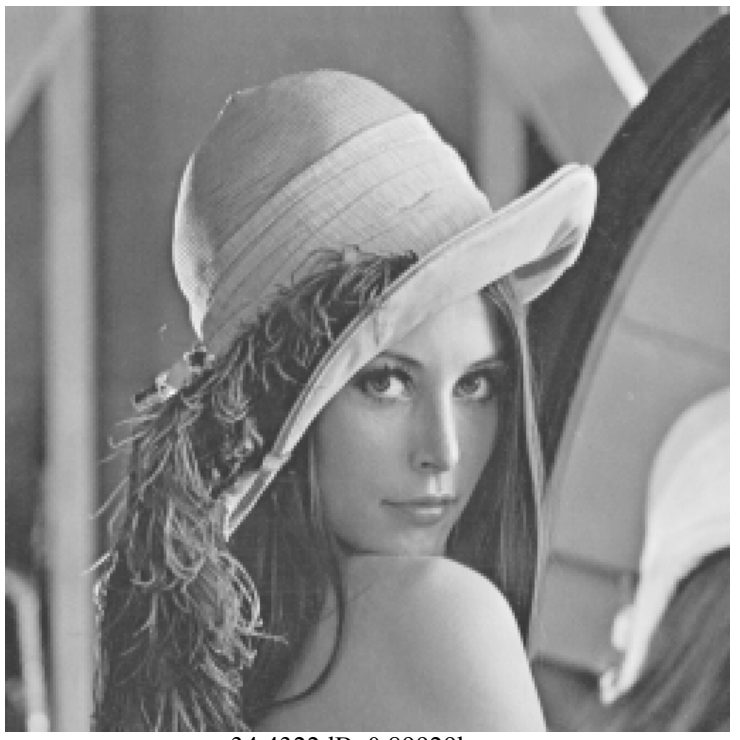

$34.4322 \mathrm{~dB}, 0.89929 \mathrm{bpp}$

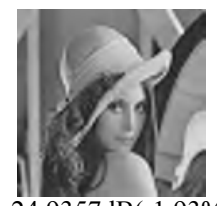

$24.9357 \mathrm{~dB}(-1.93 \%), 0.07388 \mathrm{bpp}(-42.04 \%)$

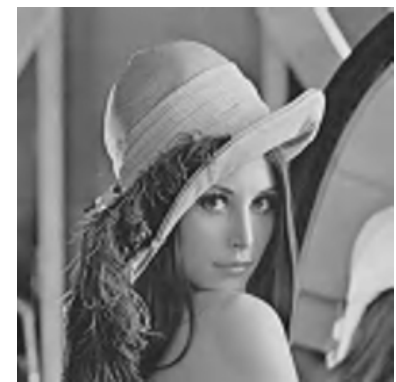

$28.8936 \mathrm{~dB}(-1.45 \%), 0.21960 \mathrm{bpp}(-39.83 \%)$

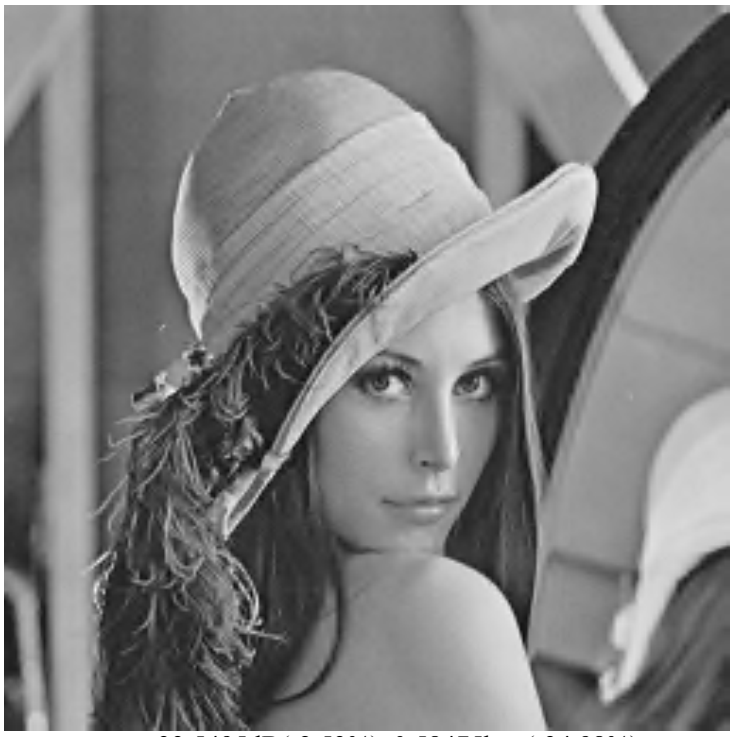

$33.5495 \mathrm{~dB}(-2.53 \%), 0.58475 \mathrm{bpp}(-34.98 \%)$

Fig. 6. Reconstructed image "Lena". $1^{\text {st }}$ column: resolution scalable; $2^{\text {nd }}$ column: regularity and resolution scalable. $1^{\text {st }}$ row: at resolution scale $4 ; 2^{\text {nd }}$ row: at resolution scale $3 ; 3^{\text {rd }}$ row: at resolution scale 2 . The quantization step is equal to 6 

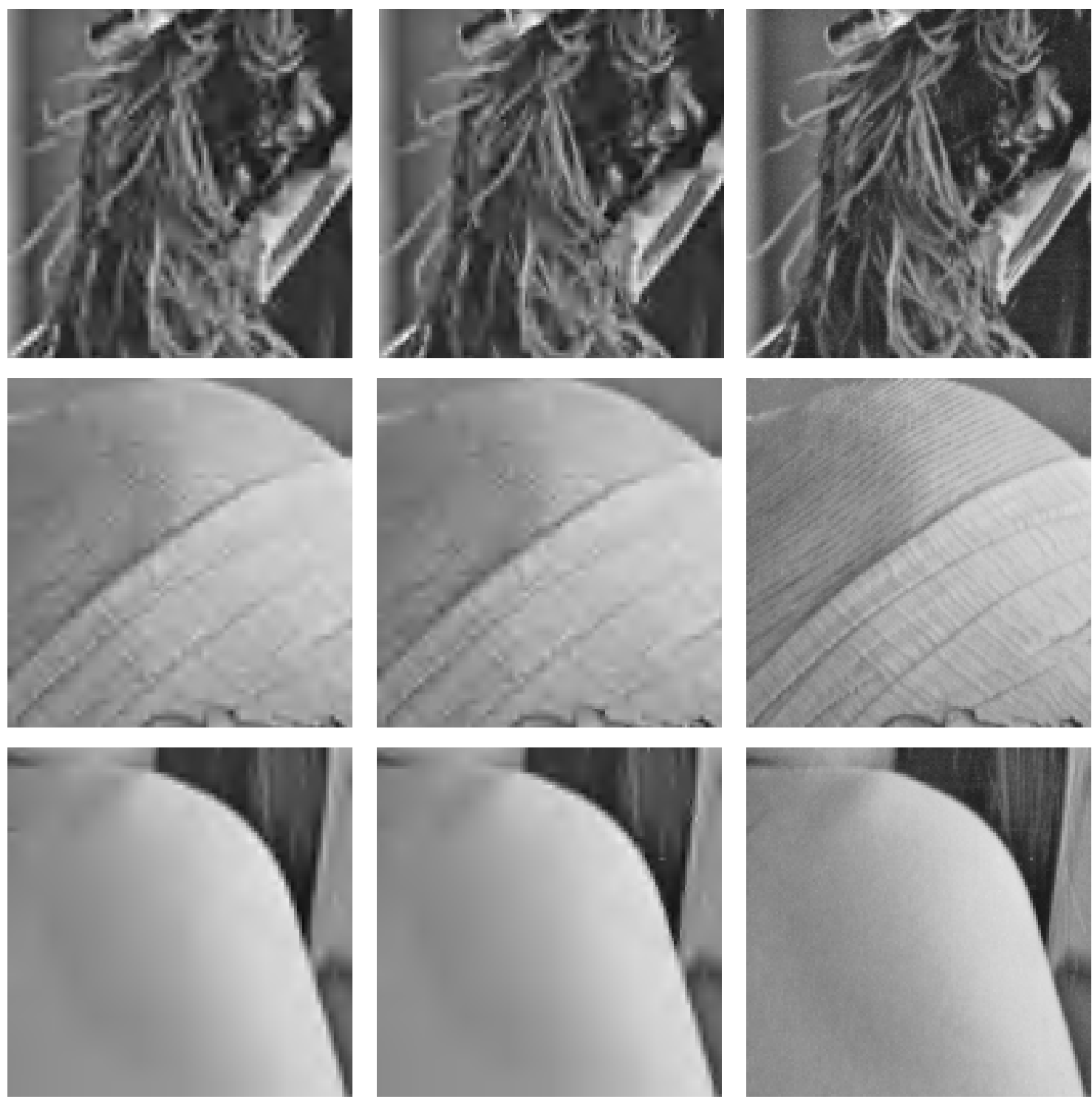

Fig. 7. Different parts of the reconstructed image "Lena" at resolution scale 2 with the highest precision. The quantization step is $2.1^{\text {st }}$ column: resolution scalable only $(34.5795 \mathrm{~dB}, 1.49017 \mathrm{bpp}) ; 2^{\text {nd }}$ column: resolution and regularity scalable $(33.5911 \mathrm{~dB}, 0.83072 \mathrm{bpp}) ; 3^{\text {rd }}$ column: original image at $3.25668 \mathrm{bpp}$. The proposed algorithm can achieve $2.86 \%$ and $44.25 \%$ reduction in PSNR and bit rate, respectively. 
20 C. Y. F. Ho, T. C. Hsung, D. P. K. Lun, B. W. K. Ling, P. K. S. Tam and W. C. Siu

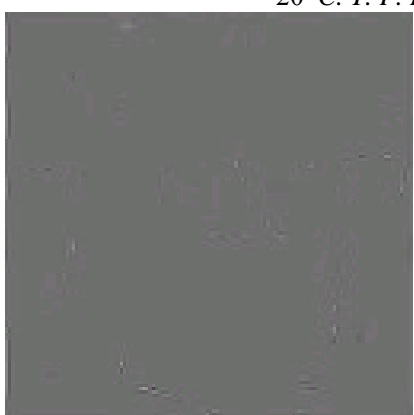

$0.0120 \mathrm{bpp}$

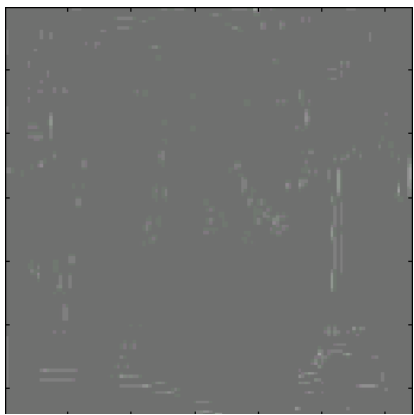

$0.0120 \mathrm{bpp}$

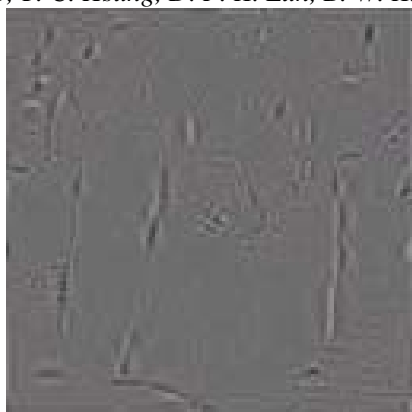

$0.2227 \mathrm{bpp}$

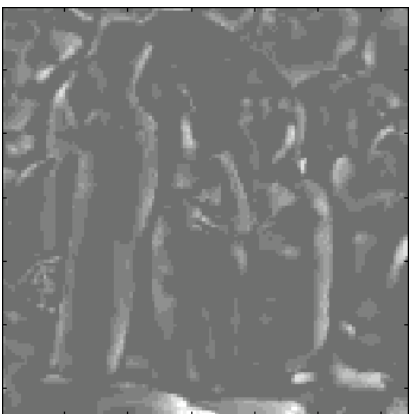

$0.2203 \mathrm{bpp}$

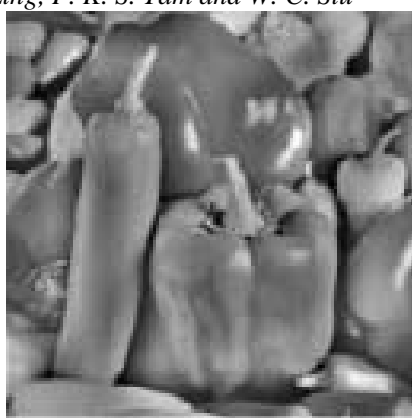

$0.3004 \mathrm{bpp}$

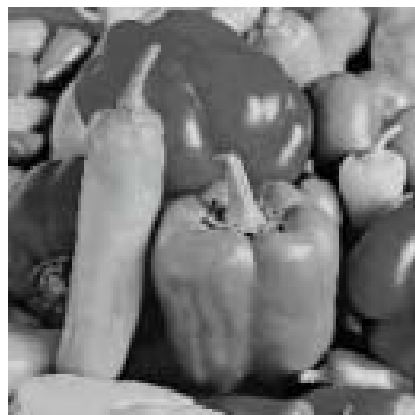

$0.2990 \mathrm{bpp}$

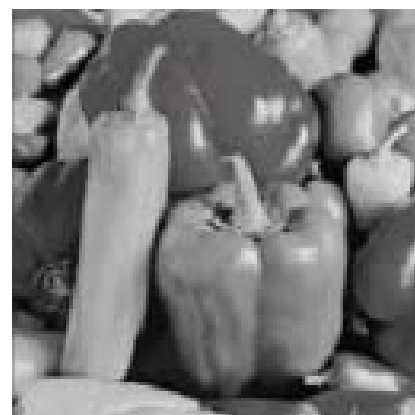

$0.3274 \mathrm{bpp}$

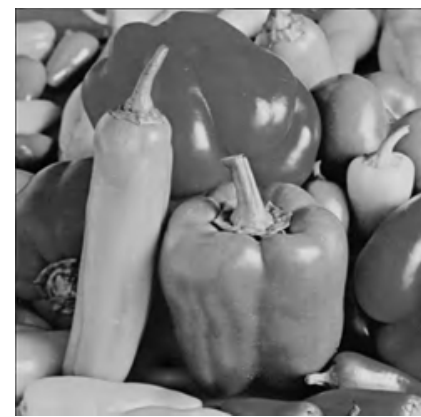

$0.3235 \mathrm{bpp}$

Fig. 8a. The reconstructed images "Pepper" at various regularity levels (second row) and the results of the

edge-oriented progressive coding algorithm ${ }^{5}$ (first row).

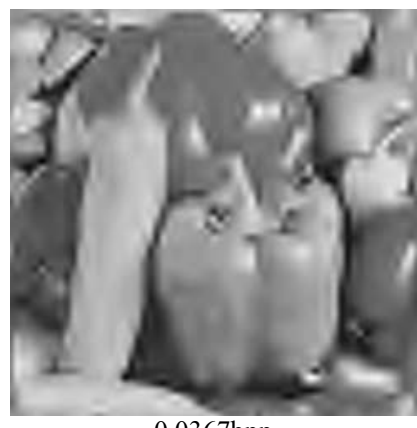

$0.0367 \mathrm{bpp}$

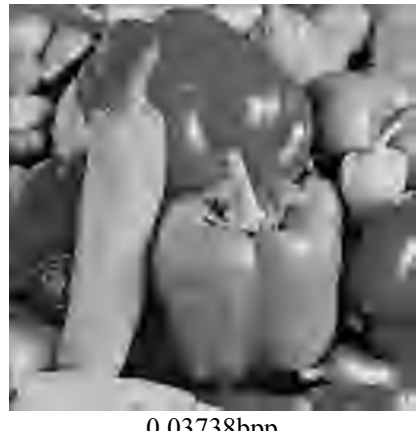

$0.03738 \mathrm{bpp}$

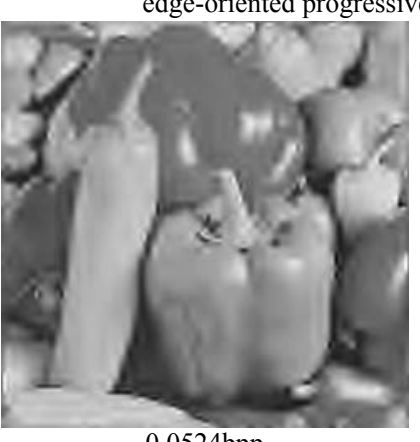

$0.0524 \mathrm{bpp}$

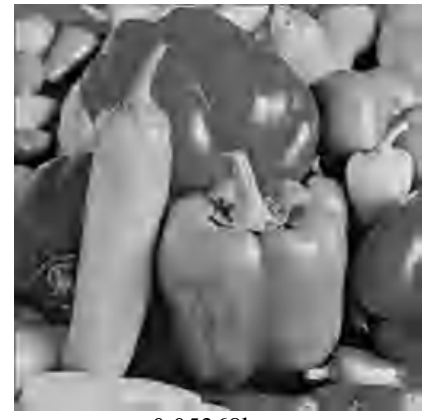

$0.05368 \mathrm{bpp}$

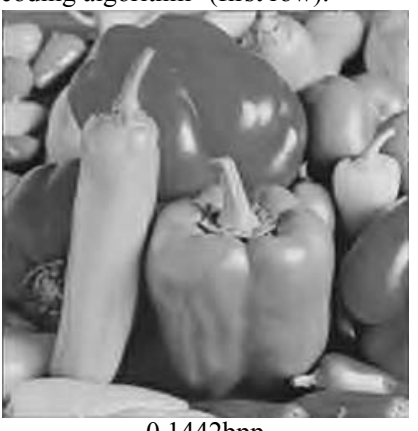

$0.1442 \mathrm{bpp}$

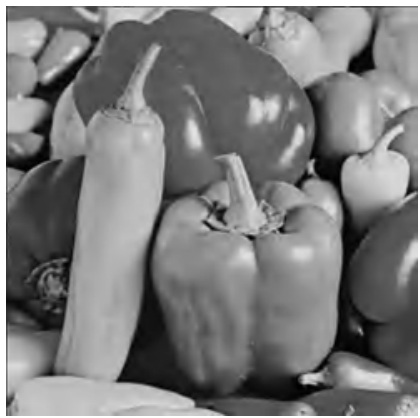

$0.1443 \mathrm{bpp}$

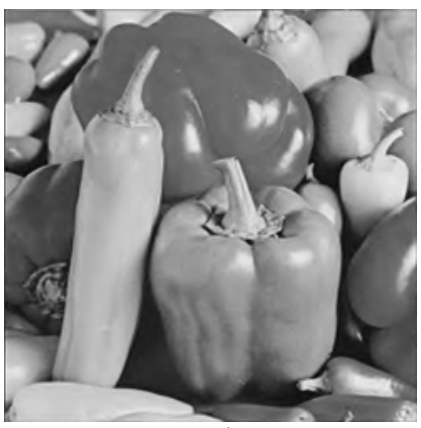

$0.2808 \mathrm{bpp}$

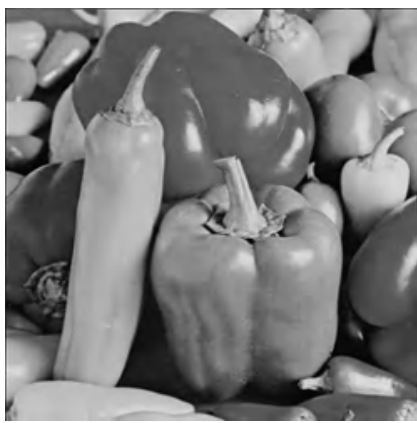

$0.2822 \mathrm{bpp}$

Fig. 8 b. The reconstructed images "Pepper" at various regularity levels (second row) and the results of the surface-oriented progressive coding algorithm ${ }^{7}$ (first row). 


\section{Conclusion}

In computer vision or pattern recognition systems, multiscale edges of an image are often detected by oriented wavelets in the undecimated domain. In this paper, we show that it can also be achieved in the decimated domain. Based on this property, we further propose an adaptive regularity scalable wavelet image coding algorithm to demonstrate the application of the estimation of the Lipschitz regularity from modulus sum from the decimated separable wavelet transform.

The proposed image coding algorithm achieves regularity scalability by selecting different decimated wavelet coefficients at different regularity levels. The selection is based on the interscale ratios of the wavelet transform magnitude sums over the "cones of influence' which is computed adaptively. The proposed regularity scalable coding algorithm outperforms various existing scalable coding algorithms in terms of visual perception, implementation complexity and coding efficiency. For the visual perception, since the proposed regularity scalable coding algorithm emphasizes on a particular feature of an image, it can provide a better visual perception of an image at very low bit rates. Simulation results show that the proposed regularity scalable coding algorithm is also benefited by its high coding efficiency. For the computational complexity, since only the computation of the sum of the wavelet coefficients and their interscale ratios in the decimated domain is required, the computational cost is low. On the other hand, for those edge-enhanced coding algorithms, there are high computational costs on the edge detection algorithms implemented in the undecimated domain. Another advantage of our proposed algorithm is that it can be embedded in any existing wavelet image coder, such as MPEG-4 still image codec, in which it is compatible with other scalable coding techniques, such as resolution scalability, without changing the bitstream format. In addition, similar to ref. 12 and ref. 13, it also possesses good robustness to noise by rejecting wavelet coefficients with negative Lipschitz exponents, which correspond to noise.

This scalable coding algorithm would be very useful in image browsing and retrieval applications. For image browsing application, a user may want to search through the database to find an image with particular features and minimize the number of transmitted bits. With the proposed scalable coding algorithm, which is based on Lipschitz regularity, an image becomes easier to be recognized at low bit rates and some particular features of an image will be visualized first at these low bit rates.

\section{Appendix A}

Proof of the necessary condition Eq. (8) in Theorem 1:

Since $f$ is Lipschitz $\alpha$ in $\mathrm{U}(\mathbf{v}), f$ is also Lipschitz $\alpha$ at $\mathbf{v}, \forall \mathbf{v} \in \mathrm{U}(\mathbf{v})$, by Eq. (3), there exists a polynomial $p_{\mathrm{v}}$ of degree $n>\lfloor\alpha\rfloor$ and $K>0$ such that

$$
\left|f\left(x_{1}, x_{2}\right)-p_{\mathbf{v}}\left(x_{1}, x_{2}\right)\right| \leq K\left(\left|x_{1}-v_{1}\right|^{2}+\left|x_{2}-v_{2}\right|^{2}\right)^{\frac{\alpha}{2}} .
$$




$$
\begin{gathered}
\left|\mathrm{W}_{j}^{l} f(\mathbf{u})\right|=\left|\iint_{-\infty}^{+\infty} f\left(x_{1}, x_{2}\right) \psi_{j, \mathbf{u}}^{l}\left(x_{1}, x_{2}\right) d x_{1} d x_{2}\right| \\
=\left|\iint_{-\infty}^{+\infty}\left(f\left(x_{1}, x_{2}\right)-p_{\mathbf{v}}\left(x_{1}, x_{2}\right)\right) \psi_{j, \mathbf{u}}^{l}\left(x_{1}, x_{2}\right) d x_{1} d x_{2}\right| \text {, by Eq. (4), } \\
\leq \iint_{-\infty}^{+\infty}\left|f\left(x_{1}, x_{2}\right)-p_{\mathbf{v}}\left(x_{1}, x_{2}\right)\right|\left|\psi_{j, \mathbf{u}}^{l}\left(x_{1}, x_{2}\right)\right| d x_{1} d x_{2} \\
\leq \iint_{-\infty}^{+\infty} K\left(\left|x_{1}-v_{1}\right|^{2}+\left|x_{2}-v_{2}\right|^{2}\right)^{\frac{\alpha}{2}}\left|\psi_{j, \mathbf{u}}^{l}\left(x_{1}, x_{2}\right)\right| d x_{1} d x_{2} \text {, by Eq. (A.1). }
\end{gathered}
$$

By Cauchy Schwarz inequality, $|a+b|^{r} \leq 2^{r}\left(|a|^{r}+|b|^{r}\right)$, so

$$
\left|\mathrm{W}_{j}^{l} f(\mathbf{u})\right| \leq A \iint_{-\infty}^{+\infty}\left(\left|x_{1}-v_{1}\right|^{\alpha}+\left|x_{2}-v_{2}\right|^{\alpha}\right)\left|\psi_{j, \mathbf{u}}^{l}\left(x_{1}, x_{2}\right)\right| d x_{1} d x_{2} .
$$

With the change of variables, $x_{1}^{\prime}=2^{-j} x_{1}-u_{1}$ and $x_{2}^{\prime}=2^{-j} x_{2}-u_{2}$, we have

$$
\begin{gathered}
\left|\mathrm{W}_{j}^{l} f(\mathbf{u})\right| \leq A\left(2^{j}\right)^{\alpha}\left(\iint_{-\infty}^{+\infty}\left|x_{1}^{\prime}\right|^{\alpha}\left|\psi^{l}\left(x_{1}^{\prime}, x_{2}^{\prime}\right)\right| d x_{1}^{\prime} d x_{2}^{\prime}+\iint_{-\infty}^{+\infty}\left|x_{2}^{\prime}\right|^{\alpha}\left|\psi^{l}\left(x_{1}^{\prime}, x_{2}^{\prime}\right)\right| d x_{1}^{\prime} d x_{2}^{\prime}\right. \\
\left.+\left|u_{1}-2^{-j} v_{1}\right|^{\alpha} \iint_{-\infty}^{+\infty}\left|\psi^{l}\left(x_{1}^{\prime}, x_{2}^{\prime}\right)\right| d x_{1}^{\prime} d x_{2}^{\prime}+\left|u_{2}-2^{-j} v_{2}\right|^{\alpha} \iint_{-\infty}^{+\infty}\left|\psi^{l}\left(x_{1}^{\prime}, x_{2}^{\prime}\right)\right| d x_{1}^{\prime} d x_{2}^{\prime}\right) .
\end{gathered}
$$

Since we assume that the mother wavelet $\psi(\mathbf{x})$ has $n$ vanishing moments, $\psi^{l}(\mathbf{x})$ also has $n$ vanishing moments for all $l$, and is $C^{n}$ with the derivatives having a fast decay. So by Eq. (5), we have

$$
\left|\mathrm{W}_{j}^{l} f(\mathbf{u})\right| \leq A\left(2^{j}\right)^{\alpha}\left(2+\left|u_{1}-2^{-j} v_{1}\right|+\left|u_{2}-2^{-j} v_{2}\right|\right) .
$$

For $\mathbf{u} \in \mathrm{U}(\mathbf{v})$, we can choose $\mathbf{v}=\left(2^{-j} v_{1}, 2^{-j} v_{2}\right)=\mathbf{u}$, so we have $\left|\mathrm{W}_{j}^{l} f(\mathbf{u})\right| \leq A\left(2^{j}\right)^{\alpha}$.

\section{Appendix B}

Proof of the sufficient condition Eq. (9) of Theorem 1:

To prove that $f$ is uniformly Lipschitz with $\alpha$ in $\mathrm{U}_{\delta}(\mathbf{v})$, we must verify that there exists $K>0$ such that for all $\mathbf{v} \in \mathrm{U}_{\delta}(\mathbf{v})$, we can find a polynomial $p_{\mathbf{v}}$ of degree $\lfloor\alpha\rfloor$ such that

$$
\forall \mathbf{x}, \mathbf{v} \in \mathfrak{R}^{2},\left|f\left(x_{1}, x_{2}\right)-f\left(v_{1}, v_{2}\right)\right| \leq K\left(\left|x_{1}-v_{1}\right|^{2}+\left|x_{2}-v_{2}\right|^{2}\right)^{\frac{\alpha}{2}}
$$

Case $1 \quad \mathbf{x} \notin \mathrm{U}_{\delta / 2}(\mathbf{v})$ 
Then $\left|x_{1}-v_{1}\right| \geq \delta / 2$ and $\left|x_{2}-v_{2}\right| \geq \delta / 2$. Since $f$ is bounded, Eq. (A.3) can be verified with a constant $K>0$ that depends on $\delta$.

Case $2 \quad \mathbf{x} \in \mathrm{U}_{\delta / 2}(\mathbf{v})$

Since $f$ can be decomposed in a Littlewood-Paley type sum as follows:

$$
f(\mathbf{x})=\sum_{j \rightarrow-\infty}^{+\infty} \Delta_{j}(\mathbf{x})
$$

with

$$
\Delta_{j}(\mathbf{x})=\frac{1}{\mathrm{C}_{\psi^{\prime}}} \iint_{-\infty}^{+\infty} \int_{2^{j}}^{2^{j+1}} \mathrm{~W}_{j}^{l} f(\mathbf{u}) \frac{1}{s} \psi^{l}\left(2^{-j} \mathbf{x}-\mathbf{u}\right) \frac{d s}{s^{2}} d u_{1} d u_{2},
$$

where $\mathrm{C}_{\psi^{\prime}}=\mathrm{C}_{\phi} \mathrm{C}_{\psi}$ or $\mathrm{C}_{\psi^{\prime}}=\mathrm{C}_{\psi} \mathrm{C}_{\psi}$, in which $\mathrm{C}_{\psi}=\int_{-\infty}^{+\infty} \frac{|\hat{\psi}(\omega)|^{2}}{\omega} d \omega<+\infty$ and $s=2^{j}$. Let $\mathrm{D}_{1}^{k-r} \mathrm{D}_{2}^{r} \Delta_{j}$ be the $k^{\text {th }}$ derivative of $\Delta_{j}$. To prove that $f$ is uniformly Lipschitz with $\alpha$ in $\mathrm{U}_{\delta}(\mathbf{v})$, we shall approximate $f$ with a polynomial $p_{\mathbf{v}}(\mathbf{x})=f(\mathbf{v})$ that generates the Taylor polynomial as follows:

$$
p_{\mathbf{v}}(\mathbf{x})=\sum_{j \rightarrow-\infty}^{+\infty} \sum_{k=0}^{\alpha\rfloor} \frac{\left[\left(\left(x_{1}-v_{1}\right) \mathrm{D}_{1}+\left(x_{2}-v_{2}\right) \mathrm{D}_{2}\right)^{k} \Delta_{j}\right](\mathbf{v})}{k !} .
$$

Since $\psi^{l}(\mathbf{x})$ has fast decay, by Eq. (5) and Eq. (A.2), we have

$$
\begin{aligned}
& \left|\Delta_{j}(\mathbf{x})\right| \leq \frac{1}{\mathrm{C}_{\psi}} \iint_{-\infty}^{+\infty} \int_{2^{j}}^{2^{j+1}} A s^{\alpha}\left(2+\left|u_{1}-2^{-j} v_{1}\right|^{\alpha^{\prime}}+\left|u_{2}-2^{-j} v_{2}\right|^{\alpha^{\prime}}\right) \frac{\mathrm{C}_{q}}{1+\left(2^{-j} x_{1}-u_{1}\right)^{q}+\left(2^{-j} x_{2}-u_{2}\right)^{q}} \frac{d s}{s^{2}} d u_{1} d u_{2} \\
& =K\left(2^{j}\right)^{\alpha} \iint_{-\infty}^{+\infty} \frac{2+\left|u_{1}-2^{-j} v_{1}\right|^{\alpha^{\prime}}+\left|u_{2}-2^{-j} v_{2}\right|^{\alpha^{\prime}}}{1+\left(2^{-j} x_{1}-u_{1}\right)^{q}+\left(2^{-j} x_{2}-u_{2}\right)^{q}} \frac{d u_{1} d u_{2}}{2^{j}} .
\end{aligned}
$$

By the inequality $|a-b|^{r} \leq 2^{r}\left(|a-c|^{r}+|c-b|^{r}\right)$, with $a=u, b=2^{-j} v$ and $c=2^{-j} x$, and with the change of variables, $u_{1}^{\prime}=u_{1}-2^{-j} x_{1}$ and $u_{2}^{\prime}=u_{2}-2^{-j} x_{2}$,

$$
\left|\Delta_{j}(\mathbf{x})\right| \leq K\left(2^{j}\right)^{\alpha} \iint_{-\infty}^{+\infty} \frac{2+\left|u_{1}^{\prime}\right|^{\alpha^{\prime}}+\left|u_{2}^{\prime}\right|^{\alpha^{\prime}}+\left|2^{-j}\left(x_{1}-v_{1}\right)\right|^{\alpha^{\prime}}+\left|2^{-j}\left(x_{2}-v_{2}\right)\right|^{\alpha^{\prime}}}{1+\left(u_{1}^{\prime}\right)^{q}+\left(u_{2}^{\prime}\right)^{q}} d u_{1}^{\prime} d u_{2}^{\prime}
$$

By choosing $m=\alpha^{\prime}+2$, we have, 
24 C. Y. F. Ho, T. C. Hsung, D. P. K. Lun, B. W. K. Ling, P. K. S. Tam and W. C. Siu

$$
\left|\Delta_{j}(\mathbf{x})\right| \leq K\left(2^{j}\right)^{\alpha}\left[2+2^{\alpha^{\prime}}\left(\left|2^{-j}\left(x_{1}-v_{1}\right)\right|^{\alpha^{\prime}}+\left|2^{-j}\left(x_{2}-v_{2}\right)\right| \alpha^{\alpha^{\prime}}\right)\right]
$$

Note that this is also applied to the partial derivatives of $\Delta_{j}(\mathbf{x}) \forall k \leq\lfloor\alpha\rfloor+1$, that is:

$$
\left|\mathrm{D}_{1}^{k-r} \mathrm{D}_{2}^{r} \Delta_{j}(\mathbf{x})\right| \leq K\left[\left(2^{j}\right)^{\alpha-k+r}\left(1+\left|2^{-j}\left(x_{1}-v_{1}\right)\right|^{\alpha^{\prime}}\right)+\left(2^{j}\right)^{\alpha-r}\left(1+\left|2^{-j}\left(x_{2}-v_{2}\right)\right|^{\alpha^{\prime}}\right)\right] .
$$

At $\mathbf{x}=\mathbf{v}$, it follows that $\forall k \leq\lfloor\alpha\rfloor+1$

$$
\left|\mathrm{D}_{1}^{k-r} \mathrm{D}_{2}^{r} \Delta_{j}(\mathbf{x})\right| \leq K\left[\left(2^{j}\right)^{\alpha-k+r}+\left(2^{j}\right)^{\alpha-r}\right] .
$$

Before computing $\left|f(\mathbf{x})-p_{\mathbf{v}}(\mathbf{x})\right|$ directly, we first need to check if the polynomial $p_{\mathbf{v}}$ in Eq. (A.6) has finite number of coefficients.

Case $2.1 \mathbf{u} \in \mathrm{U}(\mathbf{v})$

For $\mathbf{u} \in \mathrm{U}(\mathbf{v})$, we can choose $\mathbf{v}=\left(2^{-j} v_{1}, 2^{-j} v_{2}\right)=\mathbf{u}$, so Eq. (A.7) becomes

$$
\left|\Delta_{j}(\mathbf{x})\right| \leq K\left(2^{j}\right)^{\alpha} \iint_{-\infty}^{+\infty} \frac{1}{1+\left(2^{-j} x_{1}-u_{1}\right)^{q}+\left(2^{-j} x_{2}-u_{2}\right)^{q}} \frac{d u_{1} d u_{2}}{2^{j}} .
$$

By the fast decay with $q=\alpha^{\prime}+2$ and at $\mathbf{x}=\mathbf{v}$, and from Eq. (A.10), we have $\left|\mathrm{D}_{1}^{k-r} \mathrm{D}_{2}^{r} \Delta_{j}(\mathbf{x})\right| \leq K\left[\left(2^{j}\right)^{\alpha-k+r}+\left(2^{j}\right)^{\alpha-r}\right] \forall k \leq\lfloor\alpha\rfloor+1$ directly.

Case $2.2 \mathbf{u} \notin \mathrm{U}(\mathbf{v})$

Observing that $\left|2^{-j} x_{1}-u_{1}\right| \geq \delta / 2$ and $\left|2^{-j} x_{2}-u_{2}\right| \geq \delta / 2$ for $\mathbf{x} \in \mathrm{U}_{\delta / 2}(\mathbf{v})$, and $\alpha$ is not an integer, so $\alpha>\lfloor\alpha\rfloor$. These guarantee a fast decay of $\left|\mathrm{D}_{1}^{k-r} \mathrm{D}_{2}^{r} \psi_{j}^{l}(\mathbf{x})\right|$ in Eq. (A.5) when $2^{j}$ goes to zero. At large scales $2^{j}$, since $\left|\mathrm{W}_{j}^{l} f\right| \leq\|f\|\left\|\psi^{l}\right\|$, with the change of variables $\mathbf{u}^{\prime}=2^{-j} \mathbf{x}-\mathbf{u}$ in Eq. (A.5), we have

$$
\left|\mathrm{D}_{1}^{k-r} \mathrm{D}_{2}^{r} \Delta_{j}(\mathbf{v})\right| \leq \frac{\|f\|\left\|\psi^{l}\right\|}{\mathrm{C}_{\psi}}\left(\iint_{-\infty}^{+\infty}\left|\mathrm{D}_{1}^{k-r} \mathrm{D}_{2}^{r} \psi^{l}\left(\mathbf{u}^{\prime}\right)\right| d u_{1}^{\prime} d u_{2}^{\prime}\right) \int_{2^{j}}^{2^{j+1}} \frac{d s}{s^{\frac{3}{2}+k}},
$$

and hence $\left|\mathrm{D}_{1}^{k-r} \mathrm{D}_{2}^{r} \Delta_{j}(\mathbf{x})\right| \leq K\left[\left(2^{j}\right)^{\alpha-k+r}+\left(2^{j}\right)^{\alpha-r}\right] \quad \forall k \leq\lfloor\alpha\rfloor+1$. 
Together with Eq. (A.10), this proves that the polynomial $p_{\mathrm{v}}$ defined in Eq. (A.6) has coefficients that are finite. Now, with the Littlewood-Paley decomposition, we can compute $\left|f(\mathbf{x})-p_{\mathbf{v}}(\mathbf{x})\right|$ directly as follows:

$$
\left|f(\mathbf{x})-p_{\mathbf{v}}(\mathbf{x})\right|=\left|\sum_{j \rightarrow-\infty}^{+\infty}\left(\Delta_{j}\left(x_{1}, x_{2}\right)-\sum_{k=0}^{\lfloor\alpha\rfloor} \frac{\left[\left(\left(x_{1}-v_{1}\right) \mathrm{D}_{1}+\left(x_{2}-v_{2}\right) \mathrm{D}_{2}\right)^{k} \Delta_{j}\right]\left(v_{1}, v_{2}\right)}{k !}\right)\right| \text {. }
$$

The sum over scales is divided into two parts such that $2^{J} \geq|\mathbf{x}-\mathbf{v}| \geq 2^{J-1}$. The first part considers the case when $j \geq J$, while the second part considers the case when $j<J$.

For $j \geq J$ :

Like Eq. (2), we can also use the classical Taylor theorem to show that the absolute difference between $\Delta_{j}$ and its Taylor expansion is bounded, denotes

$$
\begin{aligned}
& \mathrm{I} \equiv \sum_{j=J}^{+\infty}\left|\Delta_{j}(\mathbf{x})-\sum_{k=0}^{\lfloor\alpha\rfloor} \frac{\left[\left(\left(x_{1}-v_{1}\right) \mathrm{D}_{1}+\left(x_{2}-v_{2}\right) \mathrm{D}_{2}\right)^{k} \Delta_{j}\right](\mathbf{v})}{k !}\right| \\
& \leq \sum_{j=J}^{+\infty}\left|\frac{\sup _{\mathbf{u} \in \mathrm{U}(\mathbf{v})}\left[\left(\left(x_{1}-v_{1}\right) \mathrm{D}_{1}+\left(x_{2}-v_{2}\right) \mathrm{D}_{2}\right)^{\lfloor\alpha\rfloor+1} \Delta_{j}\right](\mathbf{u}) \mid}{(\lfloor\rfloor+1) !}\right| \\
& \leq \frac{1}{(\alpha\rfloor+1) !} \sum_{r=0}^{\lfloor\alpha+1} C_{r}^{\lfloor\alpha\rfloor+1}\left(x_{1}-v_{1}\right)^{\lfloor\alpha++1-r}\left(x_{2}-v_{2}\right)^{r} \sum_{j=J}^{+\infty}\left(\sup _{\mathbf{u} \in \mathrm{U}(\mathbf{v})}\left|\mathrm{D}_{1}^{\lfloor\alpha\rfloor+1-r} \mathrm{D}_{2}^{r} \Delta_{j}(\mathbf{u})\right|\right) .
\end{aligned}
$$

Inserting Eq. (A.9) yields

$$
\mathrm{I} \leq K \sum_{r=0}^{\lfloor\alpha\rfloor+1} C_{r}^{\lfloor\alpha\rfloor+1}\left(x_{1}-v_{1}\right)^{\lfloor\alpha+1-r}\left(x_{2}-v_{2}\right)^{r} \sum_{j=J}^{r+\infty}\left(\left(2^{-j}\right)^{\alpha\rfloor+1-r-\alpha}\left|2^{-j}\left(x_{1}-v_{1}\right)\right|^{\alpha^{\prime}}+\left(2^{-j}\right)^{r-\alpha}\left|2^{-j}\left(x_{2}-v_{2}\right)\right|^{\alpha^{\prime}}\right)
$$

and since $2^{J} \geq\left|x_{i}-v_{i}\right| \geq 2^{J-1}$ for $i=1,2$, we get $\mathrm{I} \leq K\left(\left|x_{1}-v_{1}\right|^{2}+\left|x_{2}-v_{2}\right|^{2}\right)^{\frac{\alpha}{2}}$.

For $j<J$ : denote

$$
\begin{array}{r}
\mathrm{II} \equiv \sum_{j \rightarrow-\infty}^{J-1}\left|\left(\Delta_{j}(\mathbf{x})-\sum_{k=0}^{\lfloor\alpha\rfloor} \frac{\left[\left(\left(x_{1}-v_{1}\right) \mathrm{D}_{1}+\left(x_{2}-v_{2}\right) \mathrm{D}_{2}\right)^{k} \Delta_{j}\right](\mathbf{v})}{k !}\right)\right| \\
\leq \sum_{j \rightarrow-\infty}^{J-1}\left|\Delta_{j}(\mathbf{x})\right|+\sum_{j \rightarrow-\infty}^{J-1} \mid \sum_{k=0}^{\lfloor\alpha\rfloor\left[\left(\left(x_{1}-v_{1}\right) \mathrm{D}_{1}+\left(x_{2}-v_{2}\right) \mathrm{D}_{2}\right)^{k} \Delta_{j}\right](\mathbf{v}) \mid .} \\
k !
\end{array}
$$


By Eq. (A.8), the first term

$$
\begin{aligned}
\sum_{j \rightarrow-\infty}^{J-1}\left|\Delta_{j}(\mathbf{x})\right| \leq & K \sum_{j \rightarrow-\infty}^{J-1}\left(2^{j}\right)^{\alpha}\left[2+2^{\alpha^{\prime}}\left(\left|2^{-j}\left(x_{1}-v_{1}\right)\right|^{\alpha^{\prime}}+\left|2^{-j}\left(x_{2}-v_{2}\right)\right|^{\alpha^{\prime}}\right)\right] \\
& \leq K\left(2^{J \alpha}+2^{J\left(\alpha-\alpha^{\prime}\right)}\left(\left|x_{1}-v_{1}\right|^{\alpha^{\prime}}+\left|x_{2}-v_{2}\right|^{\alpha^{\prime}}\right)\right) .
\end{aligned}
$$

Also, by Eq. (A.10), the second term is bounded. And since $2^{J} \geq\left|x_{i}-v_{i}\right| \geq 2^{J-1}$ for $i=1,2$, we get $\quad \mathrm{II} \leq K\left(\left|x_{1}-v_{1}\right|^{2}+\left|x_{2}-v_{2}\right|^{2}\right)^{\frac{\alpha}{2}}$. As a result, we have $|f(\mathbf{x})-f(\mathbf{v})| \leq \mathrm{I}+\mathrm{II} \leq K\left(\left|x_{1}-v_{1}\right|^{2}+\left|x_{2}-v_{2}\right|^{2}\right)^{\frac{\alpha}{2}}$, which proves that $f$ is uniformly Lipschitz $\alpha$ in $\mathrm{U}_{\delta}(\mathbf{v})$.

\section{Acknowledgements}

The work described in this paper was supported by The Hong Kong Polytechnic University partially under project numbers B-Q395 and G-YD26 from the Hong Kong Research Grant Committee.

\section{References}

1. J. Vass, J. Yao, A. Joshi and K. Palaniappan, Interactive image retrieval over the internet, Proceedings of IEEE Symposium on Reliable Distributed Systems, 461-466 (1998).

2. J. M. Shapiro, Embedded image coding using zerotrees of wavelet coefficients, IEEE Transactions on Signal Processing, 41, 3445-3462 (1993).

3. A. Said and W. A. Pearlman, A new, fast and efficient image codec based on set partitioning in hierarchical trees, IEEE Transactions on Circuits and Systems for Video Technology, 6, 243-250 (1996).

4. D. Taubman, High performance scalable image compression with EBCOT, IEEE Transactions on Image Processing, 9, 1158-1170 (2000).

5. N. F. Law and W. C. Siu, Progressive image coding based on visually important features, Proceedings of International Conference on Image Processing, 362-366 (1999).

6. D. Schilling and P. Cosman, Edge-enhanced image coding for low bit rates, Proceedings of International Conference on Image Processing, 741-751 (1998).

7. N. Liu, Scalable wavelet image coding with photoclinometry criterion, submitted to International Conference on Information, Communications and Signal Processing (2001).

8. J. Canny, A computational approach to edge detection, IEEE Transactions on Pattern Analysis and Machine Intelligence, 36, 961-1005 (1986).

9. S. Mallat and S. Zhong, Characterization of signals from multiscale edges, IEEE Transactions on Pattern Analysis and Machine Intelligence, 14, 710-732 (1992).

10. S. Mallat and W. Hwang, Singularity detection and processing with wavelets, IEEE Transactions on Information Theory, 38, 617-643 (1992).

11. S. Mallat, A wavelet tour of signal processing, Academic Press $2^{\text {nd }}$ Editions, 1999.

12. T. C. Hsung, D. P. K. Lun and W. C. Siu, Denoising by singularity detection, IEEE Transactions on Image Processing, 47, 3139-3144 (1999). 
13. T. C. Hsung, T. C. L. Chan, D. P. K. Lun and D. D. Feng, Embedded singularity detection zerotree wavelet coding, Proceedings of International Conference on Image Processing, 274-278 (1999).

14. W. K. Carey, D. B. Chuang and S. S. Hemami, Regularity-preserving image interpolation, IEEE Transactions on Image Processing, 8, 1293-1297 (1999).

15. E. P. Simencelli, W. T. Freeman, E. H. Adelson and D. J. Heeger, Shiftable multiscale transforms, IEEE Transactions on Information Theory, 38, 587-607 (1992).

\section{Photo and Bibliography}

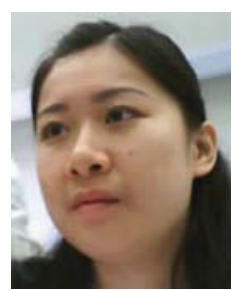

Yuk-Fan Ho received the B.Eng. (Hons) degree from the department of Electrical and Electronic Engineering, the Hong Kong University of Science and Technology in 2000, and the MPhil. Degree from the department of Electronic and Information Engineering, the Hong Kong Polytechnic University, in 2003. She is pursuing her PhD study at the Queen Mary, University of London. Her research interests include image processing, filter banks and wavelets theory, functional inequality constrained optimization problems, symbolic dynamics as well as fuzzy and impulsive control theory.

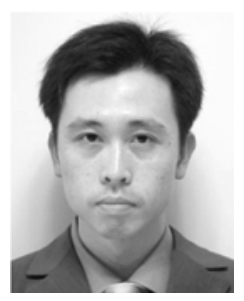

Tai-Chiu Hsung (M'93) received the B. Eng. (Hons.) and Ph.D. degrees in electronic and information engineering in 1993 and 1998, respectively, from the Hong Kong Polytechnic University, Hong Kong. In 1999, he joined the Hong Kong Polytechnic University as a Research Fellow. His research interests include wavelet theory and applications, tomography, and fast algorithms. Dr. Hsung is a member of IET.

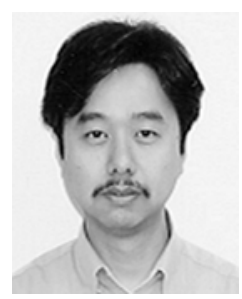

Daniel Pak-Kong Lun (M'91) received the B.Sc. (Hons.) degree from the University of Essex, Essex, U.K., and the Ph.D. degree from the Hong Kong Polytechnic University, Hung Hom, Hong Kong, in 1988 and 1991, respectively. He is currently an Associate Head of the Department of Electronic and Information Engineering, Hong Kong Polytechnic University. His research interests include digital signal processing, wavelets, multimedia technology, and Internet technology. Dr. Lun was the Secretary, Treasurer, ViceChairman, and Chairman of the IEEE Hong Kong Chapter of Signal Processing in 1994, 1995-1996., 1997-1998, 1999-2000, respectively. He was the Finance Chair of 2003 IEEE International Conference on Acoustics, Speech and Signal Processing, held in Hong Kong, in April 2003. He is a Chartered Engineer and a Corporate member of IEE. 


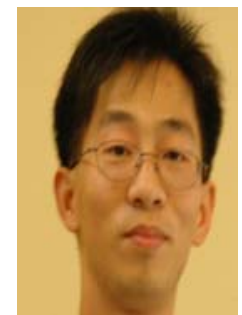

Wing-Kuen Ling received the B.Eng. (Hons) and M.Phil. degrees from the department of Electrical and Electronic Engineering, the Hong Kong University of Science and Technology, in 1997 and 2000, respectively, and the Ph.D. degree in the department of Electronic and Information Engineering from the Hong Kong Polytechnic University in 2003. In 2004, he joined the King's College London as a Lecturer. He has served as a technical committee member and an organizer of several IEEE international conferences, as well as a guest editor of International Journal of circuits, Systems and Signal Processing. His research interests include image processing, filter banks and wavelets theory, functional inequality constrained optimization problems, symbolic dynamics as well as fuzzy and impulsive control theory.

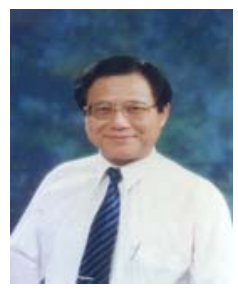

Peter Kwong-Shun Tam (S'74-M'76) received the B.E., M.E., and Ph.D. degrees from the University of Newcastle, Newcastle, Australia, in 1971, 1973, and 1976, respectively, all in electrical engineering. From 1967 to 1980, he held a number of industrial and academic positions in Australia. In 1980, he joined the Hong Kong Polytechnic University, Kowloon, Hong Kong, as a Senior Lecturer, where he is currently an Associate Professor in the Department of Electronic and Information Engineering. His research interests include signal processing, automatic control, fuzzy systems, and neural networks. $\mathrm{He}$ has participated in the organization of a number of symposiums and conferences.

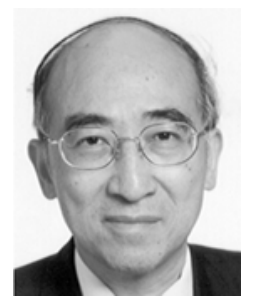

Wan-Chi Siu received the Associateship from the Hong Kong Polytechnic University and the M.Phil. degree from the Chinese University of Hong Kong in 1975 and 1977, respectively, and the Ph.D. degree from Imperial College of Science, Technology, and Medicine, London, U.K., in October 1984. He was with the Chinese University of Hong Kong as a Tutor and later as an Engineer between 1975 and 1980. He then joined the Hong Kong Polytechnic University as a Lecturer in 1980. He was promoted to Senior Lecturer, Principle Lecturer, and Reader in 1985, 1987, and 1990, respectively, and has been Chair Professor in the Department of Electronic and Information Engineering since 1992. He was Head of Department of Electronic and Information Engineering and subsequently Dean of the Engineering Faculty between 1992 and 2002. he is currently the Director of the Centre fro Multimedia Signal Processing. He has published over 250 research papers, over 120 of which appeared in international journals, such as IEEE Transactions on Signal Processing and is an editor of the recent book Multimedia Information Retrieval and Management (Springer, 2003). His research interests include digital signal processing, fast computational algorithms, transforms, wavelets, image and video coding, and computational aspects of pattern recognition. Dr. Siu was a Guest Editor, Associate Editor, and Member of the Editorial Board of the IEEE Transactions on Circuits and Systems-II, Pattern Recognition, the Journal of VLSI Signal Processing Systems for Signal, Image and Video Technology, 
the EURASIP Journal on Applied Signal Processing, in addition to other journals. He has been a keynote speaker for many international conferences, including IEEE PCM2002 (Taiwan, R.O.C.) and the IEEE 2003 ICNNP (Nanjing, China). He has held the position of General Chair or Technical Program Chair of many international conferences, including IEEE Society sponsored flagship conferences such as ISCAS'1997 and ICASSP'2003. Between 1991 and 1995, he was a member of the Physical Sciences and Engineering Panel of the Research Grants Council (RGC), Hong Kong Government, and in 1994 he chaired the first Engineering and Information Technology Panel of the Research Assessment Exercise (RAE) to assess the research quality of 19 Cost Centers (departments) from all universities in Hong Kong. He has received many awards, including the Distinguished Presenter Award (1997), the IEEE Third Millennium Medal (2000), the Best Teacher Award (2003), the Outstanding Award in Research (2003), the Plaque for Exceptional Leadership from IEEE SPCB (2003), and the Honorable Mention Winner Award from Pattern Recognition (2004). 